\title{
An Efficient FPGA Implementation of Optimized Anisotropic Diffusion Filtering of Images
}

\author{
Chandrajit Pal, ${ }^{1}$ Avik Kotal, ${ }^{2}$ Asit Samanta, ${ }^{1}$ Amlan Chakrabarti, ${ }^{1}$ and Ranjan Ghosh ${ }^{3}$ \\ ${ }^{1}$ A. K. Choudhury School of Information Technology, University of Calcutta, JD-2, Sector III, Salt Lake City, Kolkata 700098, India \\ ${ }^{2}$ Department of Applied Optics and Photonics, University of Calcutta, JD-2, Sector III, Salt Lake City, Kolkata 700098, India \\ ${ }^{3}$ Institute of Radio Physics and Electronics, University of Calcutta, JD-2, Sector III, Salt Lake City, Kolkata 700098, India
}

Correspondence should be addressed to Chandrajit Pal; palchandrajit@gmail.com

Received 11 September 2015; Revised 18 January 2016; Accepted 28 March 2016

Academic Editor: John Kalomiros

Copyright (C) 2016 Chandrajit Pal et al. This is an open access article distributed under the Creative Commons Attribution License, which permits unrestricted use, distribution, and reproduction in any medium, provided the original work is properly cited.

\begin{abstract}
Digital image processing is an exciting area of research with a variety of applications including medical, surveillance security systems, defence, and space applications. Noise removal as a preprocessing step helps to improve the performance of the signal processing algorithms, thereby enhancing image quality. Anisotropic diffusion filtering proposed by Perona and Malik can be used as an edge-preserving smoother, removing high-frequency components of images without blurring their edges. In this paper, we present the FPGA implementation of an edge-preserving anisotropic diffusion filter for digital images. The designed architecture completely replaced the convolution operation and implemented the same using simple arithmetic subtraction of the neighboring intensities within a kernel, preceded by multiple operations in parallel within the kernel. To improve the image reconstruction quality, the diffusion coefficient parameter, responsible for controlling the filtering process, has been properly analyzed. Its signal behavior has been studied by subsequently scaling and differentiating the signal. The hardware implementation of the proposed design shows better performance in terms of reconstruction quality and accelerated performance with respect to its software implementation. It also reduces computation, power consumption, and resource utilization with respect to other related works.
\end{abstract}

\section{Introduction}

Image denoising is often employed as a preprocessing step in various applications like medical imaging, microscopy, and remote sensing. It helps to reduce speckles in the image and preserves edge information leading to higher image quality for further information processing [1]. Normal smoothing operations using low-pass filtering do not take into account intensity variations within an image and hence blurring occurs. Anisotropic diffusion filter performs edge-preserving smoothing and is a popular technique for image denoising [2]. Anisotropic diffusion filtering follows an iterative process and it requires a fairly large amount of computations to compute each successive denoised image version after every iteration. This process is continued until a sufficient degree of smoothing is obtained. However, a proper selection of parameters as well as complexity reduction of the algorithm can make it simple. Various edge-preserving denoising filters do exist targeting various applications according to the cost, power, and performance requirements. However, as a case study, we have undertaken to optimize the anisotropic diffusion algorithm and design an efficient hardware equivalent to the diffusion filter that can be applied to embedded imaging systems.

Traditional digital signal processors are microprocessors designed to perform a special purpose. They are well suited to algorithmic-intensive tasks but are limited in performance by clock rate and the sequential nature of their internal design, limiting their maximum number of operations per unit time. A solution to this increasing complexity of DSP (Digital Signal Processing) implementations (e.g., digital filter design for multimedia applications) came with the introduction of FPGA technology. This serves as a means to combine and concentrate discrete memory and logic, enabling higher integration, higher performance, and increased flexibility with their massively parallel structures. FPGA contains a uniform array of configurable logic blocks (CLBs) [3-5], memory, and DSP slices, along with other elements [6]. Most machine 
vision algorithms are dominated by low and intermediate level image processing operations, many of which are inherently parallel. This makes them amenable to parallel hardware implementation on an FPGA [7], which have the potential to significantly accelerate the image processing component of a machine vision system.

\section{Related Works}

A lot of research can be found on the requirements and challenges of designing digital image processing algorithms using reconfigurable hardware $[3,8]$. In [1], the authors have designed an optimized architecture capable of processing real-time ultrasound images for speckle reduction using anisotropic diffusion. The architecture has been optimized in both software and hardware. A prototype of the speckle reducing anisotropic diffusion (SRAD) algorithm on a Virtex-4 FPGA has been designed and tested. It achieves realtime processing of $128 \times 128$ video sequences at $30 \mathrm{fps}$ as well as $320 \times 240$ pixels with a video rate speed of $30 \mathrm{fps}[8,9]$. Atabany and Degenaar [10] described the architecture of splitting the data stream into multiple processing pipelines. It reduced the power consumption in contrast to the traditional spatial (pipeline) parallel processing technique. But their system partitioning architecture clearly reveals nonoptimized architecture as the $N \times N$ kernel has been repeated over each partition (complexity of which is $O\left(N^{2}\right)$ ). Moreover, their power value is completely estimated. The power measurements of very recent hardware designed filters, namely, the bilateral and the trilateral filter [11-13], have also been undertaken. In [14], the authors have introduced a novel FPGA-based implementation of 3D anisotropic diffusion filtering capable of processing intraoperative 3D images in real time making them suitable for applications like image-guided interventions. However, it did not reveal the acceleration rate achieved in hardware with respect to the software counterpart (anisotropic diffusion) and energy efficiency information as well as any filtered output image analysis. Authors in [15] have utilized the ability of Very Long Instruction Word (VLIW) processor to perform multiple operations in parallel using a low cost Texas Instruments (TI) digital signal processor (DSP) of series TMS320C64x+. However, they have used the traditional approach of $3 \times 3$ filter masks for the convolution operation used to calculate the filter gradients within the window. It increased the computation of arithmetic operations. There is also no information regarding the power consumption and energy efficiency.

We have also compared our design with the GPU implementations of anisotropic diffusion filters for 3D biomedical datasets [16]. In [16], the authors have implemented biomedical image datasets in NVIDIA's CUDA programming language to take advantage of the high computational throughput of GPU accelerators. Their results show an execution time of $0.206 \mathrm{sec}$ for a $128^{3}$ dataset for 9 iterations, that is, for a total number of $\left(128^{3} * 9\right)$ pixels where 9 is the number of iterations to receive a denoised image. However, once we consider $3 \mathrm{D}$ image information, the number of pixels increases thrice. In this scenario, we need only 0.1 seconds of execution time in FPGA platform as an approximation ratio with a much reduced MSE (Mean Square Error) of 53.67 instead of their average of 174 . The acceleration rate becomes $91 \mathrm{x}$ with respect to CPU implementation platform unlike the case in GPU with 13x. Secondly, their timing (execution) data does not include the constant cost of data transfer (cost of transferring data between main memory on the host system and the GPU's memory which is around 0.1 seconds). It measures only the runtime of the actual CUDA kernel which is an inherent drawback of GPU. This is due to the architecture which separates the memory space of the GPU from that of its controlling processor. Actually, GPU implementation takes more time to execute the same [17] due to lot of memory overhead and thread synchronization. Besides GPU implementation or customized implementations on DSP kits of Texas Instruments have got their own separate purpose of implementation.

\section{Our Approach}

The main contributions of our work are highlighted as follows:

(i) Firstly, the independent sections of the algorithm that can be executed in parallel have been identified followed by a detailed analysis of algorithm optimization. Thereafter, a complete pipeline hardware design of the parallel sections of the algorithm has been accomplished (gradient computations, diffusion coefficients, and CORDIC divisions).

(ii) Our proposed hardware design architecture completely substituted standard convolution operation [18], required for the evaluation of the intensity gradients within the mask. We used simple arithmetic subtraction to calculate the intensity gradients of the neighboring pixels within a window kernel, by computing only one arithmetic (pixel intensity subtraction) operation. The proposed operation saved 9 multiplications and 8 addition operations per convolution, respectively (in a $3 \times 3$ window).

(iii) The number of iterations, which is required during the filtering process, has been made completely adaptive.

(iv) Besides increasing the accuracy and reducing the power reduction, a huge amount of computational time has been reduced and the system has achieved constant computational complexity, that is, $O(1)$.

(v) We performed some performance analysis on the diffusion coefficient responsible for controlling the filtering process, by subsequently differentiating and scaling, which resulted in enhanced denoising and better quality of reconstruction.

(vi) Due to its low power consumption and resource utilization with respect to other implementations, the proposed system can be considered to be used in low power, battery operated portable medical devices. 


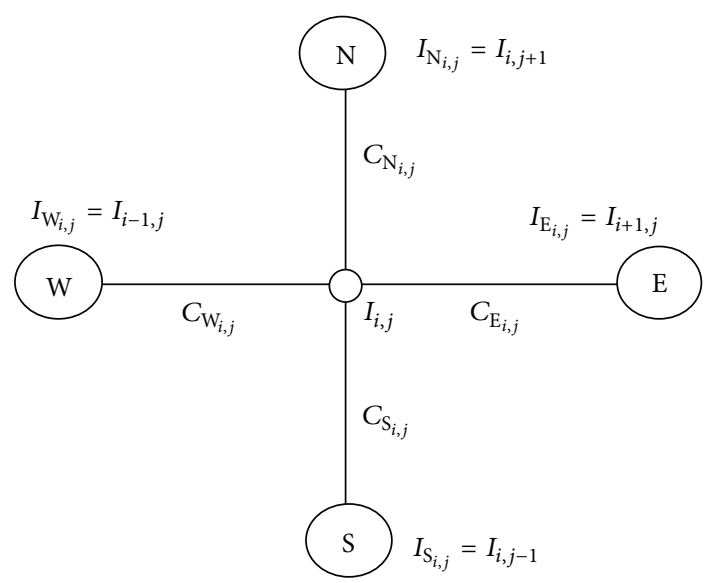

Figure 1: The structure of the discrete computational scheme for simulating the diffusion equation. The brightness values $I_{i, j}$ are associated with the nodes of a lattice and the conduction coefficients $C$ with the arcs. One node of the lattice and its four north, east, west, and south neighbors are shown [2].

The detailed description of the algorithm optimization and the hardware parallelism as well as the achieved acceleration are described in Section 5. As discussed above, in order to implement each equation, one convolution operation needs to be computed with a specified mask as per the directional gradient. Further optimization has been achieved by parallel execution of multiple operations, namely, the intensity gradient $(\nabla I)$ and the diffusion coefficients $\left(C_{n}\right)$ within the filter kernel architecture, being discussed in hardware design sections. To the best of our knowledge, this is one of the first efficient implementations of the anisotropic diffusion filtering, with respect to throughput, energy efficiency, and image quality realized in hardware.

The paper is organized as follows. Section 4 describes the algorithm background, Section 5 briefly explains the materials and methods of the approach in multiple subsections, Section 6 discusses the results, and Section 7 ends up with the conclusions and future projections.

\section{Algorithm (Background Work)}

The well known anisotropic diffusion equation is given in [2]

$$
I_{t}=\operatorname{div}(C(x, y, t) \nabla I)=C(x, y, t) \Delta I+\nabla C \cdot \nabla I
$$

where div is the divergence operator and $\nabla$ and $\Delta$, respectively, denote the gradient and Laplacian operator with respect to the space variables. $t$ denotes the time (scale) where the locations of the region boundaries appropriate for that scale are known with coordinate $(x, y)$. The anisotropic diffusion equation can be expressed as a simple numerical scheme explained as follows.

Equation (1) above can be discretized on a square lattice with vertices representing the brightness, and arcs representing the conduction coefficients, as shown in Figure 1.
An 8-nearest-neighbor discretization of the Laplacian operator can be used:

$$
\begin{gathered}
E_{\mathrm{N}}=\nabla_{\mathrm{N}} I_{i, j} \equiv I_{i-1, j}-I_{i, j}, \\
E_{\mathrm{S}}=\nabla_{\mathrm{S}} I_{i, j} \equiv I_{i+1, j}-I_{i, j}
\end{gathered}
$$

leading to

$$
\begin{aligned}
& I_{i, j}^{t+1}=I_{i, j}^{t}+\lambda\left[C_{\mathrm{N}} \cdot \nabla_{\mathrm{N}} I+C_{\mathrm{S}} \cdot \nabla_{\mathrm{S}} I+C_{\mathrm{E}} \cdot \nabla_{\mathrm{E}} I+C_{\mathrm{W}}\right. \\
& \quad \cdot \nabla_{\mathrm{W}} I+C_{\mathrm{NE}} \cdot \nabla_{\mathrm{NE}} I+C_{\mathrm{NW}} \cdot \nabla_{\mathrm{NW}} I+C_{\mathrm{SE}} \cdot \nabla_{\mathrm{SE}} I \\
& \left.\quad+C_{\mathrm{SW}} \cdot \nabla_{\mathrm{SW}} I\right]
\end{aligned}
$$

where $0 \leq \lambda \leq 1 / 4$ for the numerical scheme to be stable, $\mathrm{N}, \mathrm{S}$, $\mathrm{E}, \mathrm{W}$ are the mnemonic subscripts for north, south, east, and west, the superscripts and subscripts on the square brackets are applied to all the terms they enclose, the symbol $\nabla$, the gradient operator, indicates nearest-neighbor differences, which defines the edge estimation method, say $E$, and $t$ is the number of iterations.

Perona and Malik [2] tried with two different $g$ definitions, which controls blurring intensity according to $\|E\| ; g$ has to be a monotonically decreasing function:

$$
\begin{aligned}
& g(\|E\|)=e^{-\left(\left(\frac{\|E\|}{\kappa}\right)^{2}\right),} \\
& g(\|E\|)=\frac{1}{1+(\|E\| / \kappa)^{2}} .
\end{aligned}
$$

We define new " $C$ " to identify the conduction coefficients. The conduction coefficients are updated at every iteration as a function of the brightness gradient shown in equationarray (2). The coefficients control the amount of smoothing done at each pixel position $(x, y)$ represented as

$$
C(x, y, t)=g(\|\nabla I(x, y, t)\|) .
$$

Considering all the directions, we have

$$
\begin{gathered}
C_{\mathrm{N}_{i, j}}^{t}=g\left(\left|\nabla_{\mathrm{N}} I_{i, j}^{t}\right|\right), \\
C_{\mathrm{S}_{i, j}}^{t}=g\left(\left|\nabla_{\mathrm{S}} I_{i, j}^{t}\right|\right)
\end{gathered}
$$

If $C(x, y, t)$ is large, then $x, y$ is not a part of an edge and vice versa. Thus, substituting the value of the coefficient $\left(C_{n}\right)$ by $g()$ as shown in (6), this is performed for all the gradient directions which is finally substituted to get (3). 


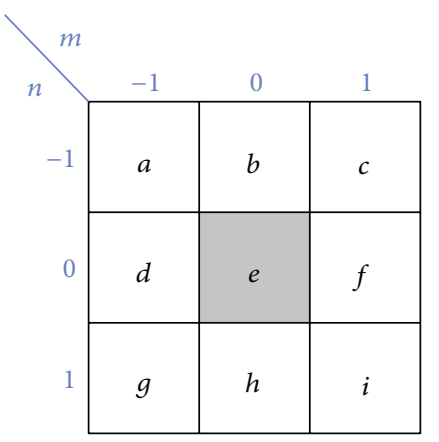

(a)

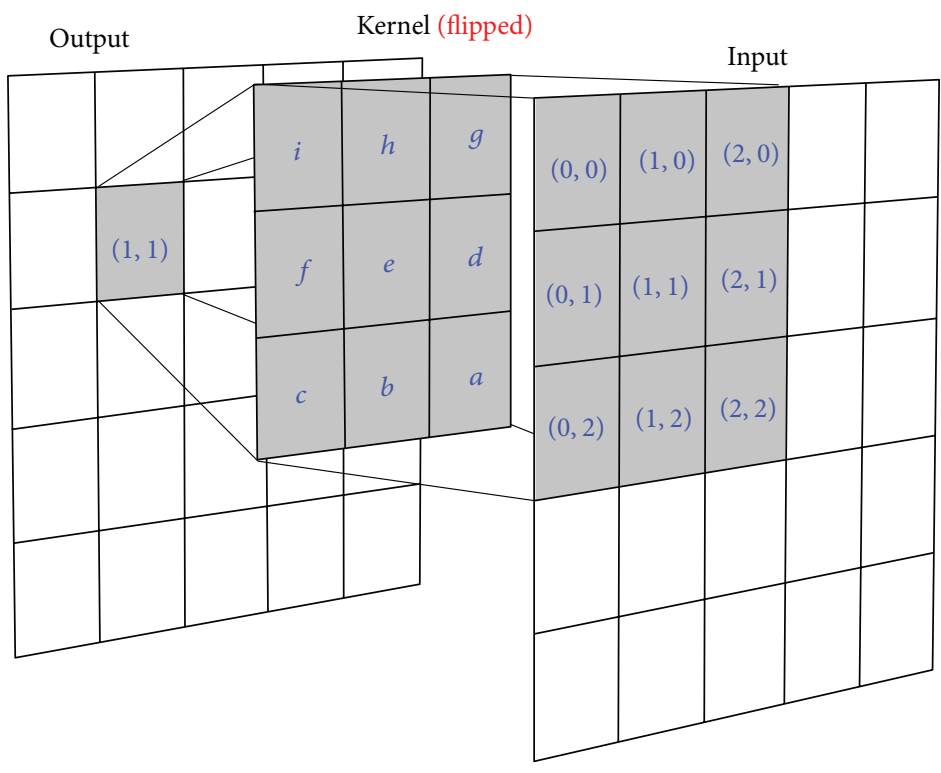

(b)

Figure 2: Convolution architecture concept. (a) $3 \times 3$ filter kernel. (b) Convolution operation (see equations (8) and (10)).

\section{Design Method and Hardware Details}

5.1. Replacing the Convolution Architecture (Proposed by Us). Consider

$$
\begin{aligned}
y[1,1]= & \sum_{j=-1}^{1} \sum_{i=-1}^{1} x[i, j] \cdot h[1-i, 1-j] \\
= & x[0,0] h[1,1]+x[1,0] h[0,1] \\
& +x[2,0] h[-1,1] \\
= & x[0,1] h[1,0]+x[1,1] h[0,0] \\
& +x[2,1] h[-1,0] \\
= & x[0,2] h[1,-1]+x[1,2] h[0,-1] \\
& +x[2,2] h[-1,-1] .
\end{aligned}
$$

Equation (7) describes a simple 2-dimensional convolution. Referring to Figure 2, we use $x$ as the input image and $h$ as the filter coefficient kernel to perform the convolution as shown in (7). Now, as a case study, substituting the value of the filter coefficient kernel (north gradient filter coefficient) is shown as follows:

$$
h_{\mathrm{N}}=\left(\begin{array}{ccc}
0 & 1 & 0 \\
0 & -1 & 0 \\
0 & 0 & 0
\end{array}\right) .
$$

In (7), we get

$$
\begin{aligned}
y[1,1] & =0+\cdots+x[1,0]-x[1,1]+\cdots+0 \\
& =x[1,0]-x[1,1] .
\end{aligned}
$$

Similarly, for south gradient filter coefficient

$$
h_{\mathrm{S}}=\left(\begin{array}{ccc}
0 & 0 & 0 \\
0 & -1 & 0 \\
0 & 1 & 0
\end{array}\right) \text {, }
$$

we get the south directional gradient as

$$
\begin{aligned}
y[1,1] & =0+\cdots+x[1,2]-x[1,1]+\cdots+0 \\
& =x[1,2]-x[1,1] .
\end{aligned}
$$

This operation is continued for all other directions. This shows that the convolution operation can be simplified down to a single arithmetic subtraction, thereby drastically reducing the number of operations, the complexity, and the hardware resources. It also enhances the speed, as discussed in the latter sections of the paper.

The gradient estimation of the algorithm for various directions is shown in equationarray (2), which was originally realized in software [19] by means of convolution of $3 \times 3$ gradient kernel sliding over the image. It consisted of 9 multiplications and 8 additions for a single convolution operation (so total of 17 operations). Therefore, our hardware realization of the convolution kernel operation (computing gradient (2)) has been substituted by a single arithmetic subtraction operation, reducing a huge amount of computation. The detailed hardware implementation is described in Section 5.5.

5.2. Adaptive Iteration (Proposed by Us). The iteration step of the filter shown in (3) needs to be manually set in the classical version of the algorithm, which was its main drawback. However, that has been made adaptive by the proposed Algorithm 1. The number of iterations completely depend upon nature of the image under consideration. 
Input: Denoised images $I_{i, j}$ at every iteration step of (3).

Comments: Referring (3).

(1) The differences between denoised output images at every iteration step is found out.

$$
I d^{t}=I_{i, j}^{t+1}-I_{i, j}^{t}
$$

(2) The difference between the maximum and minimum of the difference matrix found out in

Step (1) is computed at every iteration step.

$$
I d_{\text {diff }}^{t}=\max (I d)^{t}-\min (I d)^{t}
$$

(3) Steps (1) and (2) are continued until the condition shown below is met.

$$
\left\|I d_{\text {diff }}^{t+1}-I d_{\text {diff }}^{t}\right\|=0
$$

(4) Once the condition in Step (3) is met the execution is stopped which in turn stops the number of iteration thereby making it adaptive.

(5) Display the number of iteration thus encountered and exit.

Algorithm 1: Adaptive iteration algorithm.

5.3. In-Depth Analysis of the Diffusion Coefficient (Proposed by $U s)$. To control of the properties of the diffusion coefficient in (5) is required to analyze the signal behavior. Considering a 1-dimensional diffusion coefficient of the form shown in (12) as $1 /\left(1+x^{2}\right)$, which is a function of the gradient, we get

$$
C\left(\nabla I_{x}\right)=\frac{1}{\left(1+\nabla I_{x}^{2}\right)}
$$

where $\nabla I_{x}$ is the gradient computation shown in equationarray (2). Observing the coefficients timing variation by computing the differentiation of the coefficient $C$, we get

$$
\nabla I_{t}=\frac{\mathrm{d} C\left(\nabla I_{x}\right)}{\mathrm{d} x}=C^{\prime}\left(\nabla I_{x}\right) \cdot \nabla I_{x}^{\prime}=-\frac{2 x}{\left(x^{2}+1\right)^{2}},
$$

where $C\left(\nabla I_{x}\right)>0$ and the differentiation order may be complimented since we are interested in its timing variance:

$$
\frac{\mathrm{d}\left(\nabla I_{t}\right)}{\mathrm{d} t}=\frac{\mathrm{d}\left(\mathrm{d} C\left(\nabla I_{x}\right) / \mathrm{d} x\right)}{\mathrm{d} t}=C^{\prime \prime} \cdot \nabla I_{x}^{\prime 2}+C^{\prime} \cdot \nabla I_{x}^{\prime \prime} .
$$

Therefore, substituting the value of $C\left(\nabla I_{x}\right)$, we get

$$
\begin{aligned}
\frac{\mathrm{d}\left(\nabla I_{t}\right)}{\mathrm{d} t} & =\frac{\mathrm{d}\left[-2 \nabla I_{x} /\left(\nabla I_{x}^{2}+1\right)^{2}\right]}{\mathrm{d} x} \\
& =-\frac{2\left(\left(\nabla I_{x}^{2}+1\right)^{2}-4 \nabla I_{x}^{2}\left(\nabla I_{x}^{2}+1\right)\right)}{\left(\nabla I_{x}^{2}+1\right)^{4}} .
\end{aligned}
$$

Upon performing some algebra and rewriting, we get

$$
\frac{\mathrm{d}\left(\nabla I_{t}\right)}{\mathrm{d} t}=\frac{8 \nabla I_{x}^{2}}{\left(\nabla I_{x}^{2}+1\right)^{3}}-\frac{2}{\left(\nabla I_{x}^{2}+1\right)^{2}}=\frac{2\left(3 \nabla I_{x}^{2}-1\right)}{\left(\nabla I_{x}^{2}+1\right)^{3}} .
$$

Now, as a test case, the magnitude of the second-order derivative of the coefficient is scaled by 3 (tested on images) which changes the signal attribute as shown in Figure 3(b):

$$
\frac{\mathrm{d}\left(\nabla I_{t}\right)}{\mathrm{d} t}=\frac{6\left(3 \nabla I_{x}^{2}-1\right)}{\left(\nabla I_{x}^{2}+1\right)^{3}} .
$$

Upon solving (17), the roots appear as $\pm 1 / \sqrt{3}$. However, keeping the roots coordinate the same, the magnitude increases upon scaling as is clear from graphs (see Figure 3(b)).

So we can conclude here that the smoothing effect can be performed in a controlled manner by properly scaling the derivative of the coefficient. As a result, images with highfrequency spectrum are handled in a different way unlike their counterpart.

Since the coefficient controls the smoothing effect while denoising, it also effects the number of iterations incurred to achieve the magnitude threshold $\kappa$ in (4) for smoothing. This signal behavior of the diffusion coefficient should be very carefully handled. Proper selection of its magnitude depends upon the image selected for denoising.

5.4. Algorithm of Hardware Design Flow. The first step requires a detailed algorithmic understanding and its corresponding software implementation. Secondly, the design should be optimized after some numerical analysis (e.g., using algebraic transforms) to reduce its complexity. This is followed by the hardware design (using efficient storage schemes and adjusting fixed-point computation specifications) and its efficient and robust implementation. Finally, the overall evaluation in terms of speed, resource utilization, and image fidelity decides whether additional adjustments in the design decisions are needed (ref. Figure 4). The algorithm background has been described in the previous Section 4 .

The workflow graph shown in Figure 5 shows the basic steps of our design implementation in hardware.

5.5. Proposed Hardware Design Implementation. The noisy image is taken as an input to the FPGA through the Gateway In (see Figure 5) which defines the FPGA boundary and converts the pixel values from floating to fixed-point types for the hardware to execute. The anisotropic diffusion filtering is carried out after this. The processed pixels are then moved out through the Gateway_Out again converting the System Generator fixed-point or floating-point data type.

Figure 5 describes the abstract view of the implementation process. The core filter design has been elaborated 

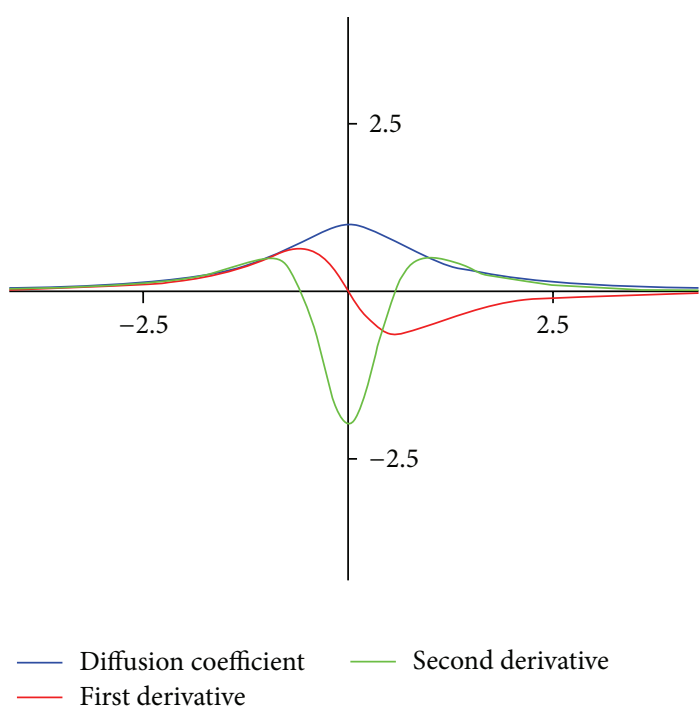

(a)

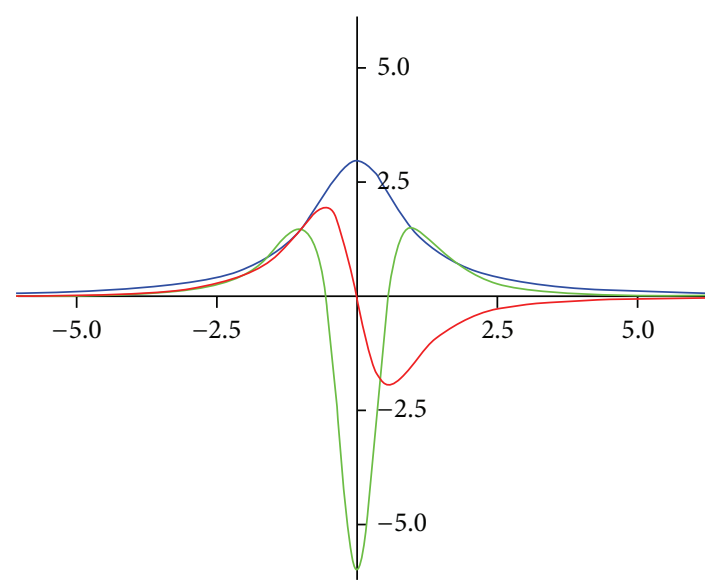

Diffusion coefficient $\quad$ - Second derivative
First derivative

(b)

FIGURE 3: Diffusion coefficient $C_{n}$ signal analysis of (12).

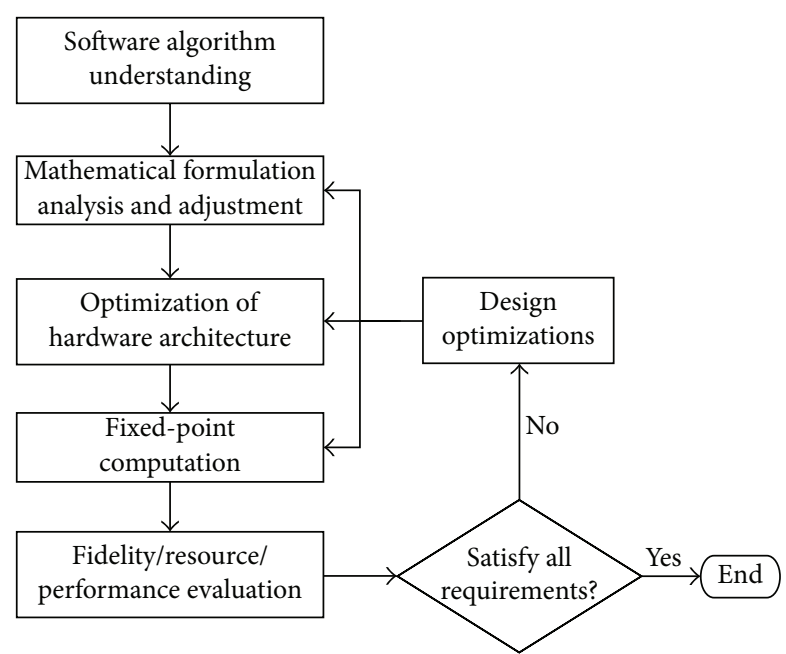

FIGURE 4: Algorithm to hardware design flow graph.

in descriptive components in a workflow modular structure shown in Figure 6. The hardware design of the corresponding algorithm is described in Figures 7-14.

Explanation of Hardware Modules as per the Workflow Diagram. Figure 7 shows the magnified view of the blue boundary block implementing equation (3) of Figure 5 (i.e., the anisotropic diffusion filtering block). Figure 7 shows the hardware design which gets fired $t$ times due to $t$ number of iterations from the script when executed.

Equation (3) has been described in words in detail in Figure 6 with iteration required to meet the necessary condition for the classical anisotropic diffusion equation. Equation (3) shows that $I_{i, j}^{t}$ gets updated at every iteration and has been realized with the hardware design in Figure 8. The green outlined box in Figure 7 has been detailed in Figure 8. The line buffer reference block buffers a sequential stream of pixels to construct 3 lines of output. Each line is delayed by 150 samples, where 150 is the length of the line. Line 1 is delayed by $(2 * 150=300)$ samples, each of the following lines are delayed by 150 fewer samples, and line 3 is a copy of the input. It is to be noted that the image under consideration used here is of resolution $150 \times 150$, and in order to properly align and buffer the streaming pixels, the line buffer should be of the same size as the image. As shown in Figure 9, $X 1$ to $X 9$ imply a chunk of 9 pixels and their corresponding positions with respect to the middle pixel $X 5$ as north $(\mathrm{N})$, south $(\mathrm{S})$, east (E), west (W), north-east (NE), north-west (NW), and so forth, as shown with a one-to-one mapping in the lower second square box.

This hardware realization of the gradient computation is achieved by a single arithmetic subtraction as described in Section 5.1.

Now, referring to this window, the difference in pixel intensities from the center position of the window to its neighbors gives its gradient as explained in equationarray (2). This difference in pixel intensities is calculated in the middle of the hardware section as shown in Figure 8. Here, $X 1$ denotes the central pixel of the processing window and the corresponding differences with pixel intensities in position $X 1, X 2, X 3, \ldots, X 9$ denote the directional gradient $(X 1-$ $X 5=$ grad_northwest, $X 2-X 5=$ grad_west $, \ldots, X 9-X 5=$ grad_southeast). The pixels are passed through the line buffers (discussed in Section 5.6) needed for proper alignment of the pixels before computation. This underlying architecture is basically a memory buffer needed to store two image lines (see Section 5.6) implemented in the FPGA as a RAM block. The deep brown outlined block in Figure 8 (from where the three out lines are coming out) contains the detailed diagram and working principle of the buffering scheme in Figure 12. 


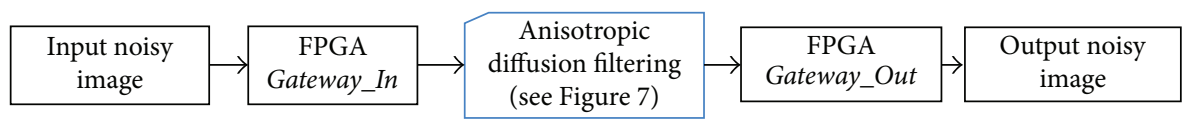

FIGURE 5: Work flow of design modules.

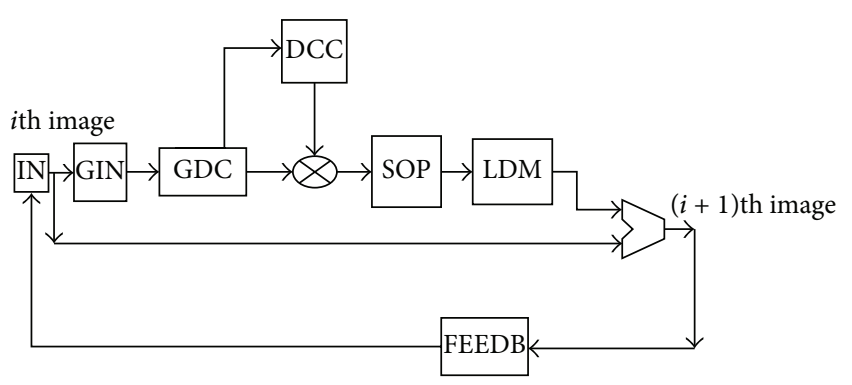

IN: input noisy image

GIN: input image is passed into the boundary of the FPGA through the Gateway_In

GDC: computing the image gradient in all eight direction within a filter window

DCC: computing the diffusion coefficient using the image gradients

Figure 6: Work flow of design module of anisotropic diffusion filter of Figure 5 in detail.

The pixels $X 1$ to $X 9$ are passed out of the buffer line blocks through various delays into the next level of circuitry as shown in Figure 8. Referring to Figure 8, the Out 1 of the line buffer block which outputs three pixels $X 7$ to $X 1$ as per Figure 9 of which $X 7$ moves out first, then $X 8$ and $X 9$ after encountering the delay blocks. Similarly, pixel data flow occurs for Out 2 and Out 3 blocks, respectively, with the pixel positions as shown from $X 1$ to $X 9$. Pixel positions at this instant of time shown in Figure 8 have been shown after encountering the buffer delays. In this model, pixel X5 denotes the center pixel and subtracting it from the remaining pixels denotes the gradient in their corresponding positions as shown in the following:

$$
\begin{aligned}
& X 1-X 5=\nabla_{\mathrm{NW}} I, \\
& X 2-X 5=\nabla_{\mathrm{W}} I, \\
& X 3-X 5=\nabla_{\mathrm{SW}} I, \\
& X 4-X 5=\nabla_{\mathrm{N}} I, \\
& X 6-X 5=\nabla_{\mathrm{S}} I, \\
& X 7-X 5=\nabla_{\mathrm{NE}} I, \\
& X 8-X 5=\nabla_{\mathrm{E}} I, \\
& X 9-X 5=\nabla_{\mathrm{SE}} I .
\end{aligned}
$$

Now, let us discuss the bottom-up design approach to make things more transparent. Referring to (3), the coefficient $C_{n}$ is defined in equationarray (6) which has been realized in hardware as shown in Figure 11 where $\|E\|$ is the intensity gradient calculating variable and $\kappa$ is the constant value 15 . So $1 / \kappa=1 / 15=0.0667$ which gets multiplied with the input gradient $\|E\|$ squared up and then added with a unitary value and the resultant becomes the divisor with 1 the dividend. Referring to the hardware design in Figure 11, the CORDIC divisor has been used to compute the division operation in (4) and the rest is quite clear. Now, Figure 10 is the hardware design of the equations $C_{n} E_{n}$ and $1 / 2 C_{n} E_{n}$ as per the individual components of (3). For the gradient north, south, east, and west, it is needed to multiply only $1 / 2$ with $C_{n} E_{n}$ and 1 for others. We have seen the coefficient computation of equationarray (6) where the input is the gradient $E_{n}=\nabla I_{n}$. This is the same input in the hardware module in Figure 10 needed to compute coefficient $C_{n}$. The output of Figure 10 is nothing but the coefficient multiplied with the gradient $E_{n}$ as shown.

The delays are injected at the intervals to properly balance (synchronize) the net propagation delays. Finally, all the output individual components of the design shown in Figure 8 are summed up and the lambda $(\lambda)$ is finally multiplied with the added results. This implementation of the line buffer is described in the next subsection.

Each component in (3), that is, $C \cdot \nabla I$, requires an initial $41-$ unit delay for each processed pixel to produce (CORDIC: $31-$ unit delay, multiplier: 3-unit delay, and register: 1-unit delay). The delay balancing is done as per the circuitry. However, this delay is encountered at first and from the next clock pulse each pixel gets executed per clock pulse since the CORDIC architecture is completely pipelined.

5.6. Efficient Storage/Buffering Schemes. Figure 12 describes the efficiency in the storage/buffering scheme. Figures 12 and 13 describe a window generator to buffer reused image intensities diminishing data redundancies. This implementation of the line buffer uses a single port RAM block with the read before write option as shown in Figure 13. Two buffer lines are used to generate eight neighborhood pixels. The length of the buffer line depends on the number of pixels in a row of an image. A FIFO structure is used to implement a $3 \times 3$ window kernel used for filtering to maximize the pipeline implementation. Leaving the first 9 clock cycles, each pixel is processed per clock cycle starting from the 10th clock cycle. The processing hardware elements never remain idle due to the buffering scheme implemented with FIFO (Figure 14). Basically, this FIFO architecture is used to implement the buffer lines.

With reference to Figure 14, it is necessary that the output of the window architecture should be vectors for pixels in the window, together with an enable which is used to inform an algorithm using the window generation unit as to when the data is ready to process. To achieve maximum performance in a relatively small space, FIFO architectural units specific to the target FPGA were used. 


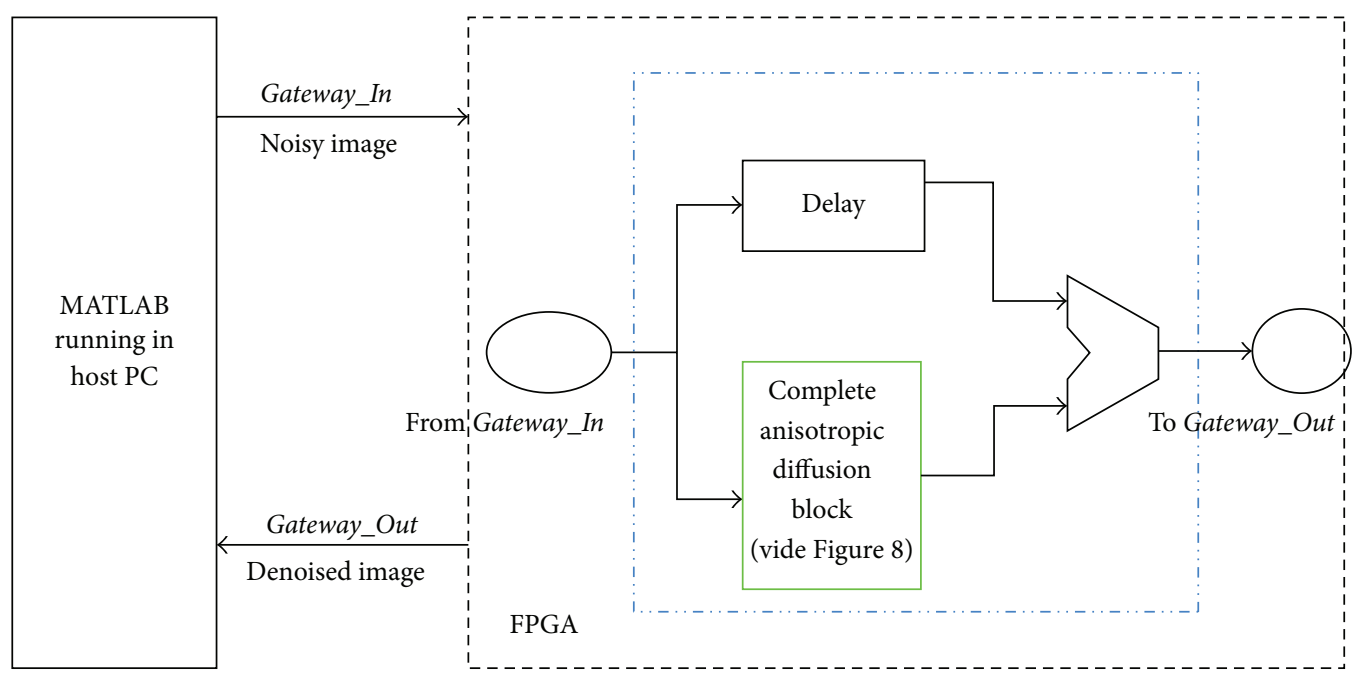

FIGURE 7: This hardware design shows a single instance of the iterative diffusion step shown in (3). The overall architecture with the pixels passing from the host PC to the FPGA platform and the processed pixels being reconstructed back to the host PC.

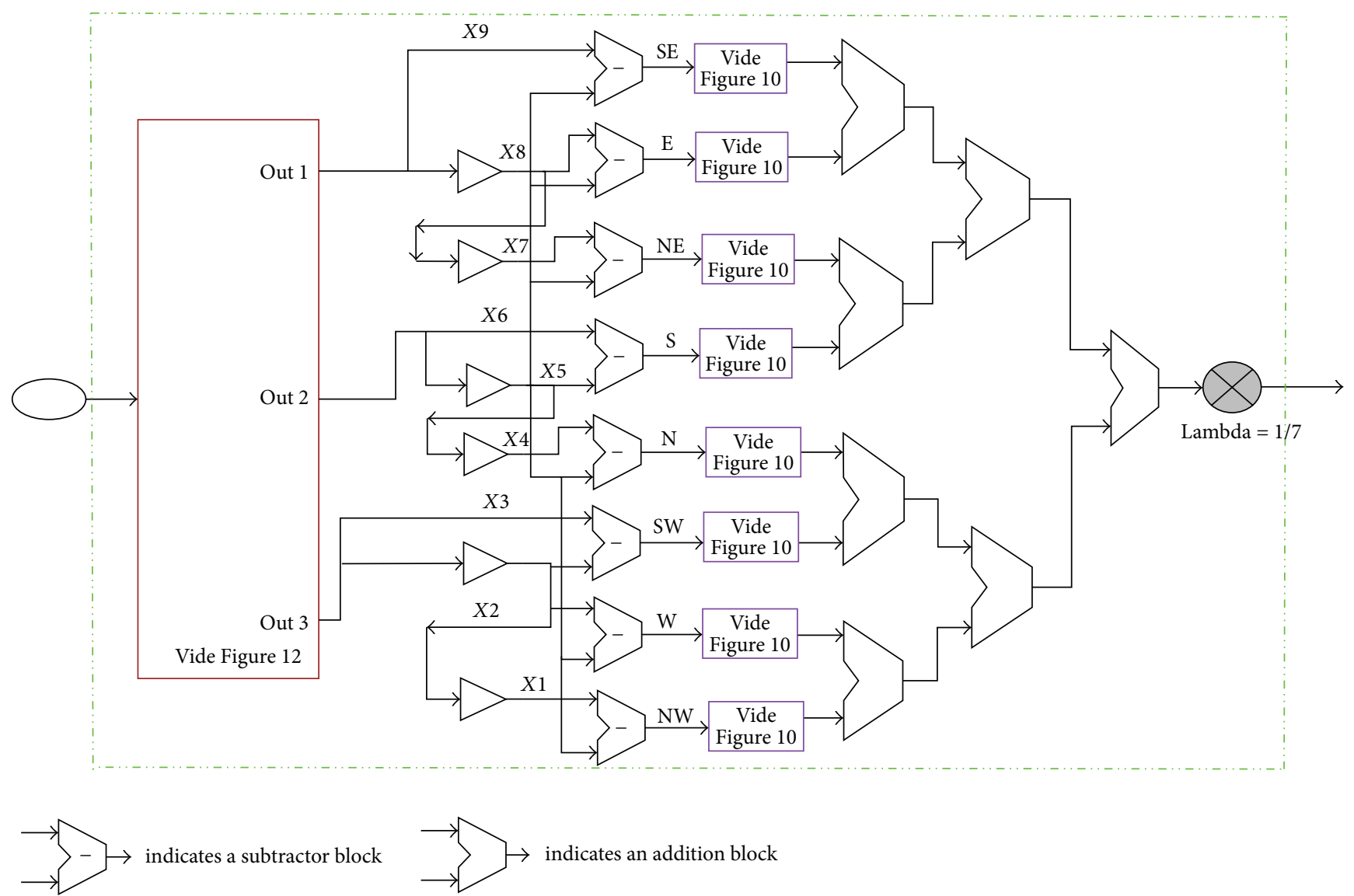

FIGURE 8: Hardware architecture of the second additive term of the RHS of (3).

\section{Results and Discussion}

In this paper, we presented an efficient architecture of the FPGA prototyped hardware design of an optimized anisotropic diffusion filtering on image. The algorithm has been successfully implemented using FPGA hardware using the System Generator platform with Intel(R) Core(TM) 2 Duo CPU T6600@ 3.2 GHz platform and Xilinx Virtex-5 LX110T OpenSPARC Evaluation Platform $(100 \mathrm{MHz})$ as well as Avnet Spartan-6 FPGA IVK. 


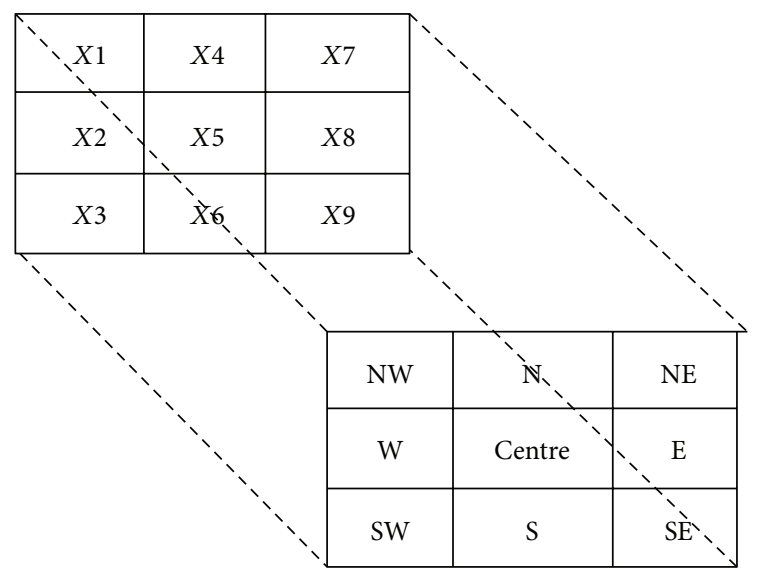

FIGURE 9: The figure is showing a section of an image and the neighborhood pixel directions with respect to the middle pixel.

Here, the hardware filter design is made using the Xilinx DSP blockset. The algorithm [2] has been analyzed and optimally implemented in hardware with a complete parallel architecture. The proposed system leads to improved acceleration and performance of the design. The throughput is increased by 12 to $33 \%$ in terms of frame rate, with respect to the existing state-of-the-art works like [20-22]. Figures 15, 16 , and 17 show the denoising performances. Figure 15 shows a denoised image of various human skeletal regions which was affected by noise. Figure 16 shows the quality comparison between the hardware and its corresponding software implementations. Figure 17 shows the various denoising filter performances. Fine texture regions have been magnified to show the achieved differences and improvement.

Figures 18 and 19 denote the accuracy measures.

With regard to data transfer requirement, there is a huge demand for fast data exchange between the image sensor and the computing platform. For example, transferring a $1024 \times 1024$ grayscale video sequence in real time requires a minimum data transfer rate of $1024 \times 1024$ pixels/frame $*$ $1 \mathrm{byte} /$ pixel $* 30 \mathrm{fps}=30 \mathrm{Mbps}$. In order to achieve this data rate, a high performance I/O interface, such as a PCI or USB, is necessary. We have used USB 2.0 (Hi-Speed USB mode) supporting $60 \mathrm{Mbps}$ data rate.

For a $512 \times 512$ image resolution, time taken to execute in software is 0.402 seconds and 0.101 seconds for $150 \times 150$ size grayscale image approximately (cf. Table 1).

Simulation activity files (SAIF) from simulation is used for accurate power analysis of a complete placed and routed design.

As already explained, the buffer line length needs to be equal to that of the image resolution. Now, as the resolution increases, the buffering time increases too. Now, it is obvious that increasing image resolution, the number of pixels to be processed in both hardware and software increases. This difference is proportionate. But what makes the difference in acceleration rate as a result of change in resolution (see Table 1) is created by the buffering scheme of the hardware. In software, the image can be read at one go unlike in hardware
TABLE 1: Runtime comparison between acceleration rates of our proposed hardware implementation of anisotropic diffusion filter for different image resolutions.

\begin{tabular}{lccc}
\hline $\begin{array}{l}\text { Image } \\
\text { resolution }\end{array}$ & $\begin{array}{c}\text { Software } \\
\text { execu- } \\
\text { tion } \\
\text { time } \\
\text { (seconds) }\end{array}$ & $\begin{array}{c}\text { Hardware } \\
\text { execution } \\
\text { time } \\
\text { (seconds) }\end{array}$ & $\begin{array}{c}\text { Accelerate } \\
\text { rate }\end{array}$ \\
\hline $150 \times 150$ & 0.101 & 0.0011 & $\mathbf{( 0 . 1 0 1 / 0 . 0 0 1 1 )}=\mathbf{9 1}$ \\
$512 \times 512$ & 0.402 & 0.0131 & $\mathbf{( 0 . 4 0 2 / 0 . 0 1 3 1 )}=\mathbf{3 0}$ \\
\hline
\end{tabular}

where the pixels need to be serialized while reading (see Figures 12 and 14).

Case 1. The image resolution used for this experiment is $150 \times$ 150, so a total of 22500 pixels. Therefore, a sum total of $(22500 * 5)=112500$ pixels have been processed for five iterations of the algorithm. Our proposed hardware architecture is such that it can process per pixel per clock pulse (duration $10 \mathrm{~ns}$ ). The clock frequency of the FPGA platform on which the experiment has been performed is $100 \mathrm{MHz}$ (period = $10 \mathrm{~ns}$ ). The Gateway In of the FPGA boundary has an unit sample period. Therefore, the total time taken to process is 22500 pixels $* 5$ iterations $* 10 \mathrm{~ns}=\mathbf{0 . 0 0 1 1}$ seconds in hardware (also has been cross-checked complying with (19)).

Whereas only in software environment the total time taken to execute in the host PC configuration mentioned above is 0.101 seconds, thus a total acceleration of $(\mathbf{0 . 1 0 1} /$ $\mathbf{0 . 0 0 1 1}=\mathbf{9 1 x}$ ) in execution speed has been achieved in FPGAin-the-loop [23] experimental setting.

Case 2. Therefore, for image resolution $512 \times 512$, the total hardware time required to process is 262144 pixels $*$ 5 iterations $* 10 \mathrm{~ns}=0.0131$ seconds (also has been crosschecked complying with (19)). Figure 20 shows that per pixel gets executed per clock cycle starting from the FPGA boundary Gateway_In to Gateway_Out.

The experiment has been implemented 10 times and the corresponding mean squared error (MSE) obtained has been averaged by 10 to get the averaged $\mathrm{MSE}_{\mathrm{av}}$, which is used to calculate the PSNR. Since the noise is random, therefore averaging is performed while computing the PSNR.

As seen from the processed images, our result resembles the exact output very closely. The difference is also clear from the difference of the PSNR and SSIM values (Table 2).

A closer look has been plotted with a one-dimensional plot shown in Figure 21, which clearly exposes the smoothing effect at every iterative step.

FPGA-in-the-loop (FIL) verification [23] has been carried out. It includes the flow of data from the outside world to move into the FPGA through its input boundary (a.k.a Gateway_In), get processed with the hardware prototype in the FPGA, and be returned back to the end user across the Gateway_Out of the FPGA boundary $[24,25]$. This approach also ensures that the algorithm will behave as expected in the real world. 
TABLE 2: Quality measures (performance metrics): SSIM (structural similarity) and PSNR (peak signal-to-noise ratio) for the experiments. For each column, the best value has been highlighted for three different noise standard deviations. Our proposed technique OAD (optimized anisotropic diffusion) shows better result except for the SSIM parameter for standard deviation, 20. Comparison has been made with different types of benchmark edge preserving denoising filters.

\begin{tabular}{|c|c|c|c|c|c|c|}
\hline \multirow{2}{*}{ Method } & \multicolumn{2}{|c|}{ Std. dev. $=12$} & \multicolumn{2}{|c|}{ Std. dev. $=15$} & \multicolumn{2}{|c|}{ Std. dev. $=20$} \\
\hline & SSIM & PSNR (dB) & SSIM & PSNR (dB) & SSIM & PSNR (dB) \\
\hline (a) $\mathrm{ADF}$ [2] & 0.9128 & 29 & 0.8729 & 27.82 & 0.8551 & 24.93 \\
\hline (b) NLM [27] & 0.9346 & 28.2 & 0.9067 & 27.29 & 0.8732 & 25.64 \\
\hline (c) $\mathrm{BF}[12]$ & 0.9277 & 27 & 0.8983 & 28.54 & 0.8809 & 24.28 \\
\hline (d) $\mathrm{TF}$ [13] & 0.8139 & 25.22 & 0.7289 & 22.87 & 0.6990 & 21.98 \\
\hline $\begin{array}{l}\text { (e) OAD } \\
\text { (Our proposed } \\
\text { optimized } \\
\text { anisotropic } \\
\text { diffusion filter) }\end{array}$ & 0.9424 & 30.01 & 0.9245 & 28.87 & 0.8621 & 25.86 \\
\hline
\end{tabular}

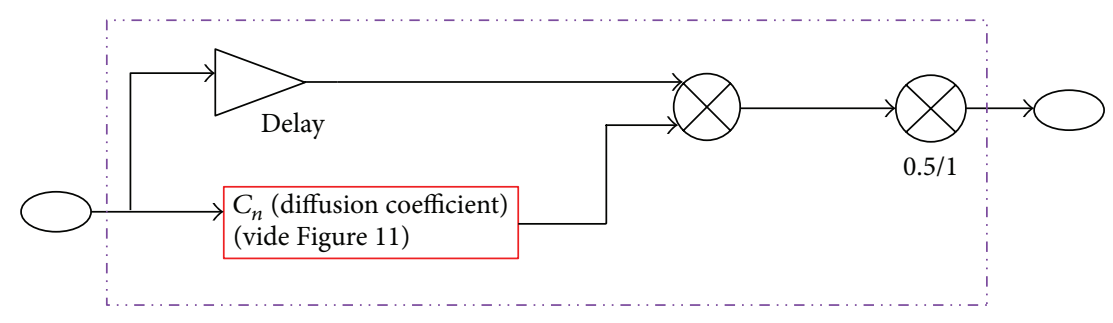

FIgURE 10: This hardware module multiplies the diffusion coefficient $C_{n}$ with the pixel gradient $\nabla I$ to produce $C_{n} \nabla I$.

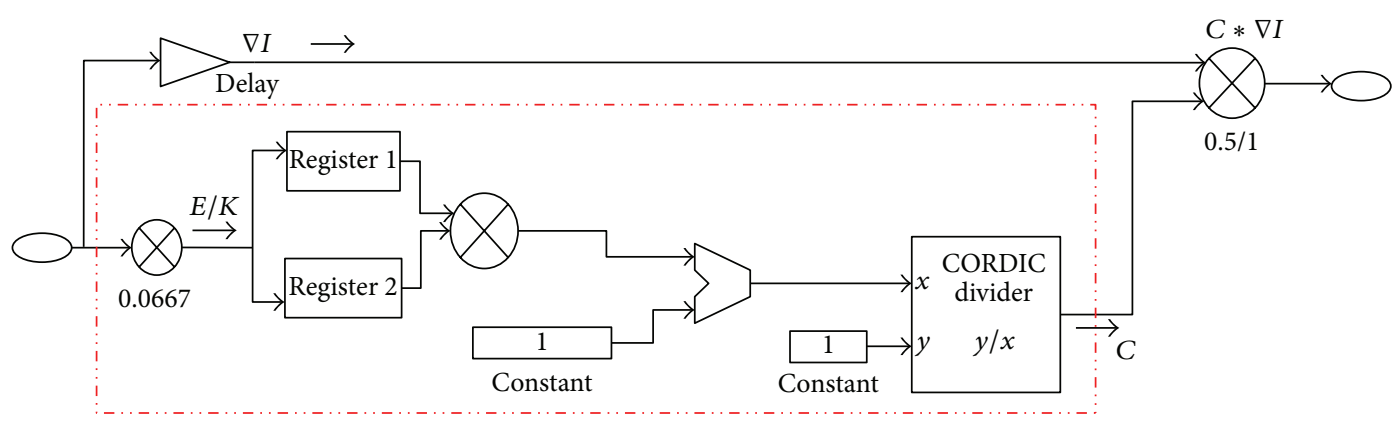

FIGURE 11: Hardware design for (5) generated for controlling the blurring intensity, that is, diffusion coefficient $\left(C_{n}\right)$.

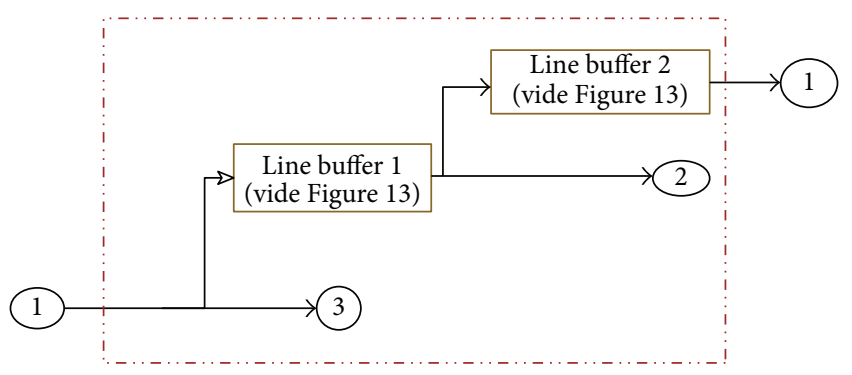

Figure 12: Hardware module showing the line buffering scheme of the pixels as described in Section 5.6 and hardware design (Section 4).

Figure 22 shows the frames per second achieved for images of various resolutions. The power measurements in our proposed method have been analyzed after the implementation phase (placement and routing) and are found to be more accurate and less than their stronger counterpart,

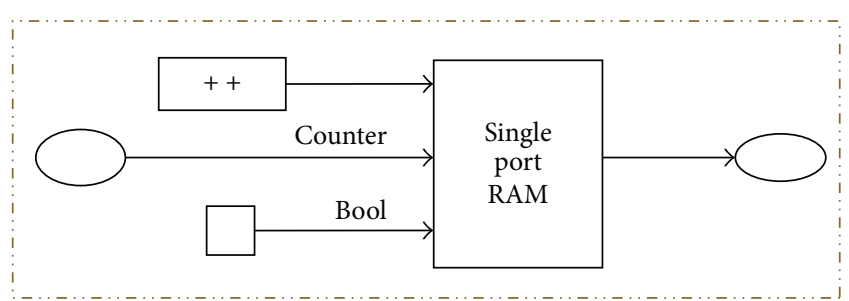

Figure 13: Hardware design within the line buffer shown in Figure 12.

namely, the hardware implementation of the bilateral and trilateral filter as shown in Table 3.

Table 4 denotes the resource utilization for both the hardware platforms for our implementation and a very strong benchmark implementation (its counterpart) of bilateral filter. It shows that a lot of acceleration has been achieved for anisotropic (cf. Table 5) with respect to its counterpart 


\begin{tabular}{|c|c|c|}
\hline w11 & w12 & w13 \\
\hline w21 & w22 & w23 \\
\hline w31 & w32 & w33 \\
\hline
\end{tabular}

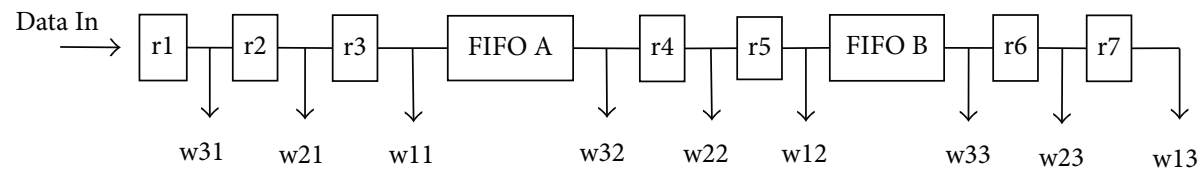

FIGURE 14: Data flow architecture of the window kernel implemented using the FIFO architecture.

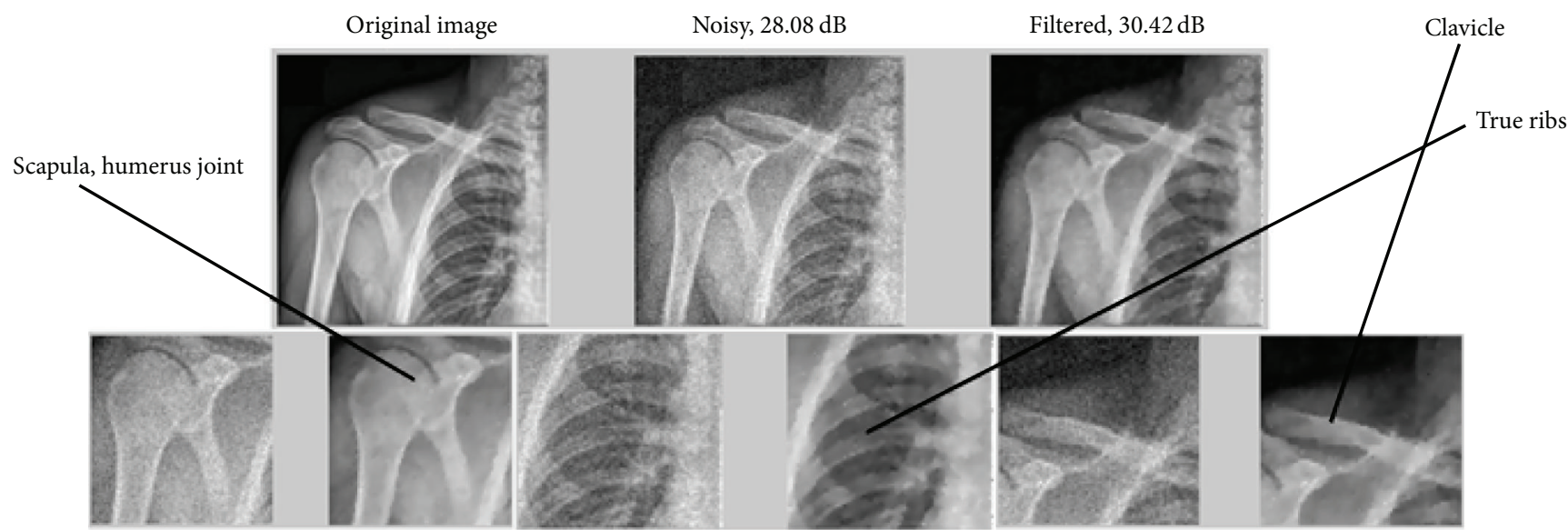

(a)

(b)

(c)

FIGURE 15: Results showing the original image, its noisy counterpart, and the denoised image and its various magnified portions of various sections of the denoised image of human skeleton. Image size $=512 \times 512$; the filter settings are as follows: Sigma $(\sigma)$ for random noise $=12$, number of iterations $=4, \lambda$ in $(3)=1 / 7$, and Kappa $(\kappa)$ in $(4)=15$. (a), (b), and (c) show the zoomed insets of the scapula-humerus joint, true rib region, and the clavicle bone, respectively.

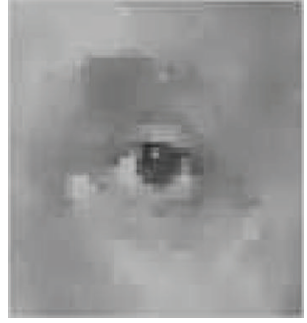

(a)

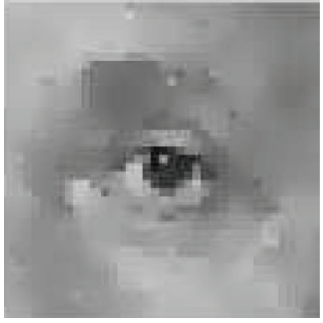

(b)

FIGURE 16: Image quality comparison between (a) FPGA implementation for the natural Einstein image (zoomed eye inset) and (b) MATLAB implementation. Filter parameters: Sigma $(\sigma)$ for random noise $=12$, number of iterations $=4, \lambda$ in $(3)=1 / 7$, and Kappa $(\kappa)$ in $(4)=15$.

bilateral at the cost of a marginal increase in percentage of resource utilization (cf. Table 5).

The complexity analysis has been compared with some of the benchmark works and is shown in Table 6.

6.1. Considerations for Real-Time Implementations. There remain some considerations while planning to implement complex image processing algorithms in real time. One such issue is to process a particular frame of a video sequence within $33 \mathrm{~ms}$ in order to process with a speed of 30 (frames per second) fps. In order to make correct design decisions, a well known standard formula is given by

$$
t_{\text {frame }}=\frac{C}{f}=\frac{\left(M \cdot N / t_{p}+\xi\right)}{n_{\text {core }}} \cdot f \leq 33 \mathrm{~ms},
$$




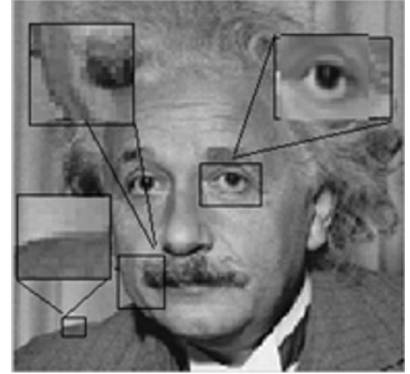

(a)

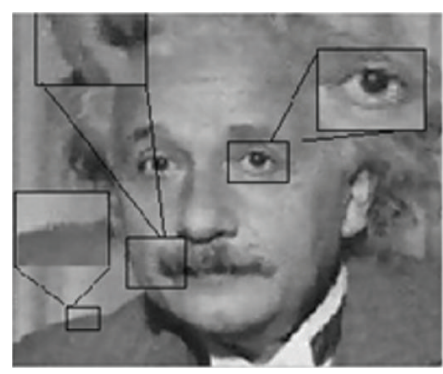

(d)

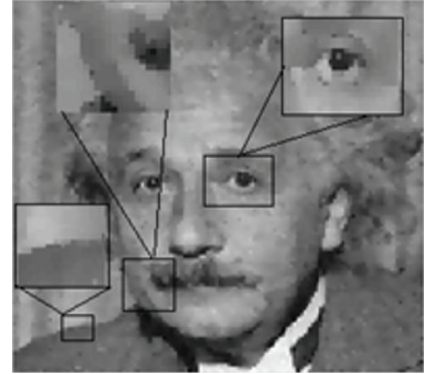

(b)

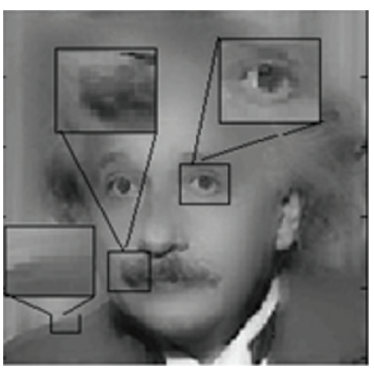

(e)

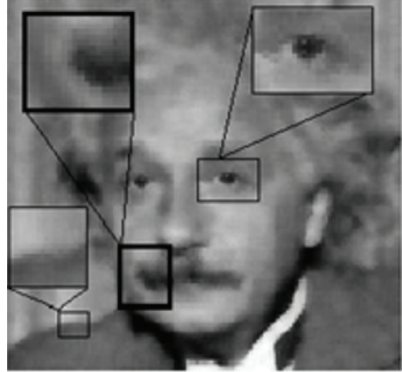

(c)

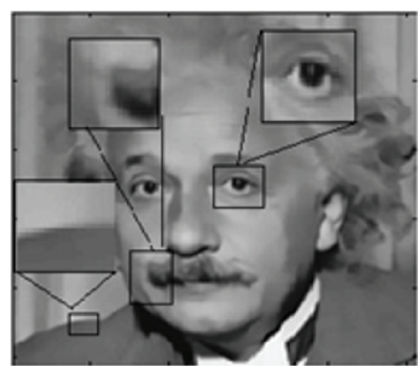

(f)

FIGURE 17: Comparison of various edge-preserving filters implemented using hardware on the natural grayscale image Einstein of size $150 \times$ 150 measured for similar number of iterations. (a) Original image. (b) Direct implementation of the anisotropic diffusion filter (software implementation). (c) Output of the bilateral filter realized using FPGA. (d) Output of nonlocal means filter. (e) Trilateral filter output. (f) Output of our implementation of optimized anisotropic diffusion filtering using a novel hardware design. Note that the fine boundary transitions in the moustache area (see zoomed insets) are clearly visible in our implementation in (f) unlike others which is clear from the visual experience. Similarly, the zoomed portions of the left eye show the clear distinctions of the lower and upper lid (also holds the contrast information); moreover, the magnified area of the neck portion also shows a sharp transition. All the comparisons should be done keeping the original image in (a) in mind as the reference image. The PSNR difference is as shown in Table 2.

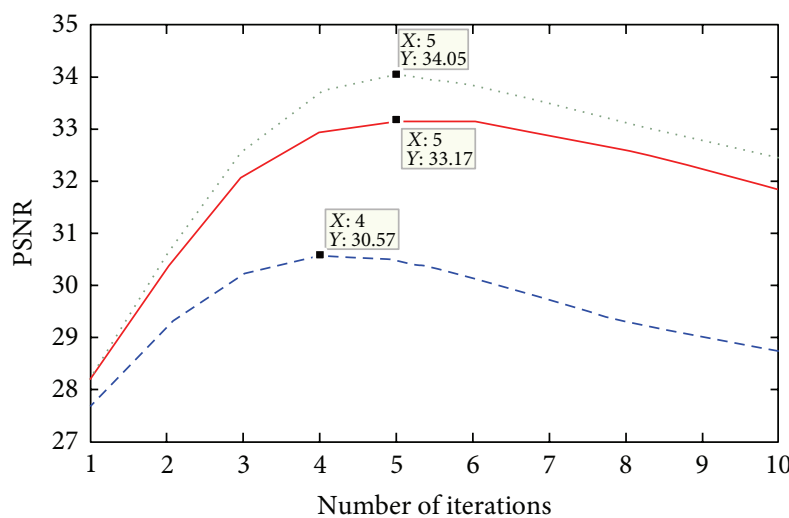

FIGURE 18: Results showing the variance of PSNR measured against the number of iterations measured for images of various resolutions. It has been found that the number of iterations ranges between 4 and 5 to attain the most denoised output close to the original. The filter settings are as follows: Sigma $(\sigma)$ for random noise $=12$, number of iterations $=4, \lambda$ in $(3)=1 / 7$, and Kappa $(\kappa)$ in $(4)=15 .(\cdots)$ : image resolution $150 \times 150$, (-): image resolution $256 \times 256$, and (- - ): image resolution $512 \times 512$. Various images with the similar resolution have been tested and the averages have been plotted with identical curves with their respective resolutions.

where $t_{\text {frame }}$ is the processing time for one frame, $C$ is the total number of clock cycles required to process one frame of $M$
TABLE 3: Power utilization for Virtex-5 OpenSPARC architecture measured for an image of resolution $150 \times 150$.

\begin{tabular}{lccc}
\hline Filter type & $\begin{array}{c}\text { Quiescent } \\
\text { power } \\
\text { (watt) }\end{array}$ & $\begin{array}{c}\text { Dynamic } \\
\text { power } \\
\text { (watt) }\end{array}$ & Total power (watt) \\
\hline $\begin{array}{l}\text { OAD (our } \\
\text { proposed) }\end{array}$ & $\mathbf{1 . 1 9 0}$ & $\mathbf{0 . 2 0 0}$ & $(1.100+0.070)=\mathbf{1 . 1 7 0}$ \\
TF [13] & 2.305 & 0.422 & $(2.305+0.422)=2.727$ \\
$\begin{array}{l}\text { BF [12] } \\
\text { Reference }\end{array}$ & 1.196 & 0.504 & $(1.196+0.504)=1.700$ \\
{$[10]$} & NA & NA & 1.240 \\
\hline
\end{tabular}

pixels, $f$ is the maximum clock frequency at which the design can run, $n_{\text {core }}$ is the number of processing units, $t_{p}$ is the pixellevel throughput with one processing unit $\left(0<t_{p}<1\right), N$ is the number of iterations in an iterative algorithm, and $\xi$ is the overhead (latency) in clock cycles for one frame [1].

So in order to process one frame, the total number of clock cycles required is given by $\left(M \cdot N / t_{p}+\xi\right)$ for a single processing unit. For $n_{\text {core }}>1$, one can employ multiple processing units.

Let us evaluate a case study applying (19) for our experiment. 


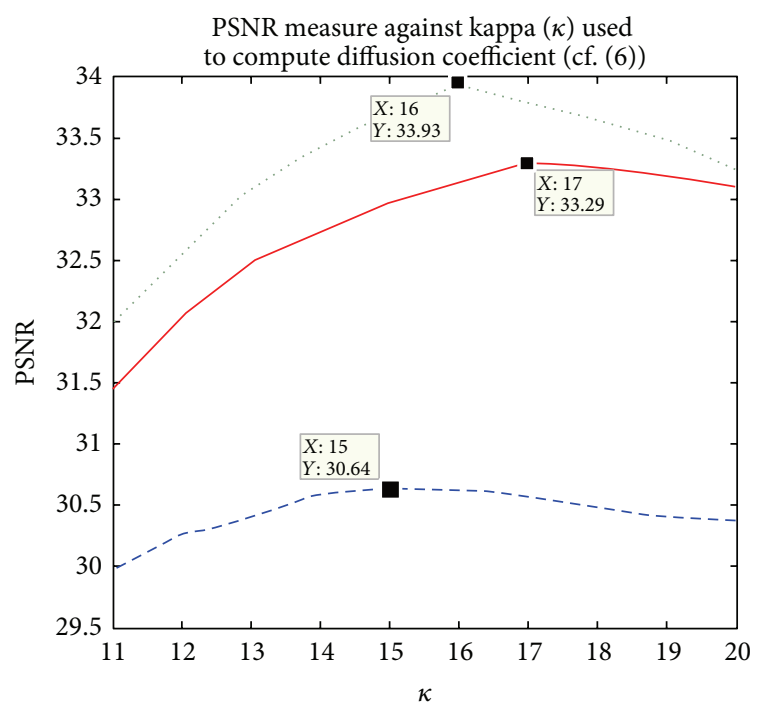

(a)

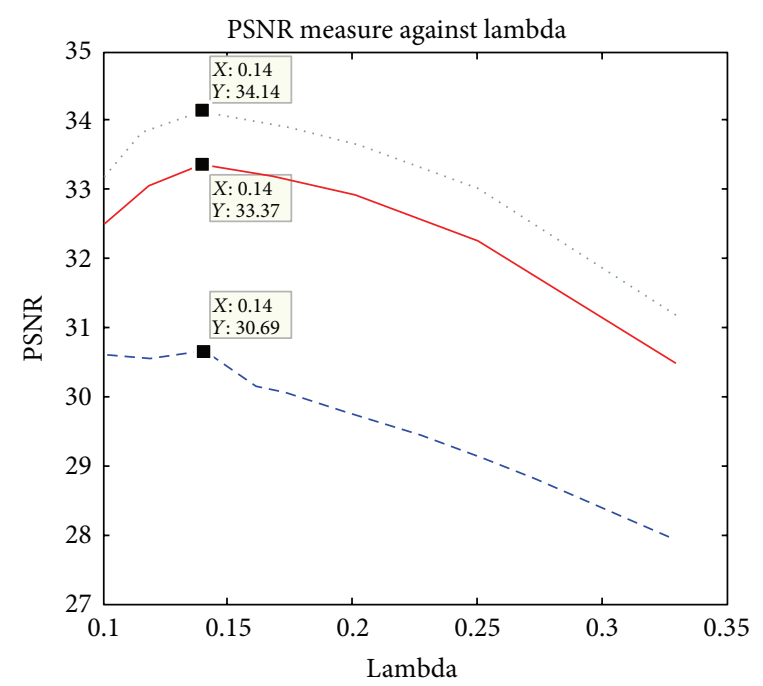

(b)

FIGURE 19: Results show two graphical plots: (a) measures the change of PSNR values against the parameter kappa $(\kappa)$ (cf. in (4)) used to compute the diffusion coefficient $\left(C_{n}\right)$, which reflects an optimum value in the range of 15 to 17 needed to obtain the denoised accuracy as shown with the pointers in the graphs. (b) shows a single value for $\lambda=1 / 7$ yields the maximum denoised output for different images of varying resolutions; the rest of the filter settings remain the same.

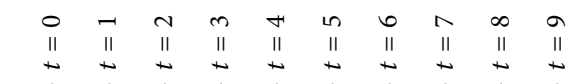

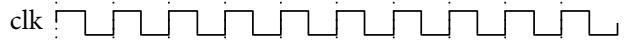

Gateway_In $1120 \times 140 \times 165 \times 160 \times 155 \times 150 \times 148 \times 148 \times 155 \times 158$

Gateway_Out $: 105 \times 108 \times 110 \times 135 \times 138 \times 139 \times 140 \times 141 \times 137 \times 128$

Line_Buffered_Output $108 \times 152 \times 155 \times 152 \times 150 \times 152 \times 155 \times 155 \times 155 \times 152$ Filtered_Output $: 1 \times 2 \times 2.5 \times 2 \times 1.2 \times 1.2 \times 1 \times 0.8 \times 0 \times 0$

FIGURE 20: Simulation results showing the time interval taken to process the image pixels. Each clock pulse duration is $10 \mathrm{~ns}$. Each pixel requires one clock pulse to process from the FPGA boundary Gateway_In to Gateway_Out together with the intermediary signal lines as probed, following the same rate (ref. Figure 7).

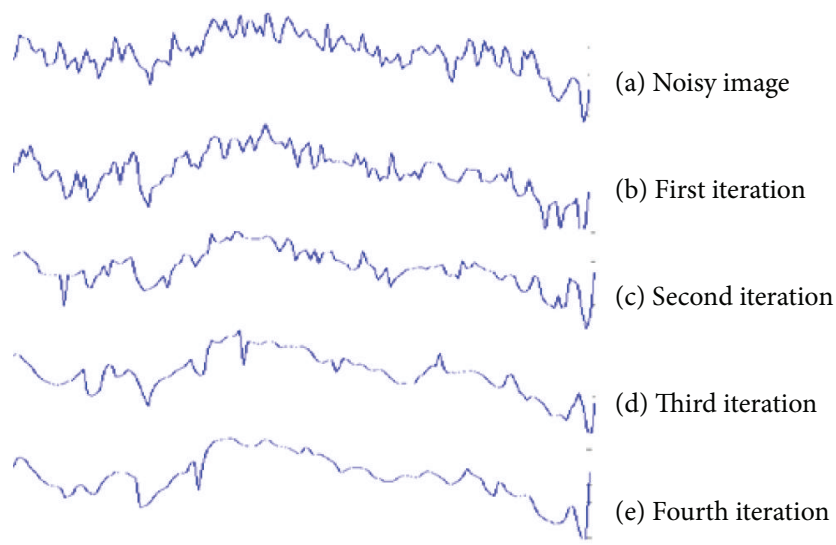

FIGURE 21: Family of 1D signals showing the plot of only one particular row of an image, the variation of which is shown at different iterations, starting from noisy to final denoised output.
For $512 \times 512$ resolution image, $M=262144, N=5$, $t_{p}=1$, that is, per pixel processed per clock pulse, $\xi=1050$, that is, the latency in clock cycle, $f=100 \mathrm{MHz}$, and $n_{\text {core }}=1$. Therefore, $t_{\text {frame }}=0.013$ seconds $=13 \mathrm{~ms} \leq 33 \mathrm{~ms}$ (i.e., much less than the minimum timer threshold required to process per frame in real-time video rate). With regard to data transfer requirement, there is a huge demand for fast data exchange between the image sensor and the computing platform. For example, transferring a $1024 \times 1024$ grayscale video sequence in real time requires a minimum data transfer rate of $1024 \times 1024$ pixels/frame $* 1$ byte/pixel $* 30 \mathrm{fps}=30 \mathrm{Mbps}$. In order to achieve this data rate, a high performance $\mathrm{I} / \mathrm{O}$ interface, such as a PCI or USB, is necessary. Our USB 2.0 ( $\mathrm{Hi}-$ Speed USB mode) supports $60 \mathrm{Mbps}$ data rate, which is just double the minimum requirement of $30 \mathrm{Mbps}$ which catered our purpose with ease.

The highlights of our approach are the following:

(i) Accuracy. We have performed our experiments on approximately 65-70 images (both natural and medical) and they are producing successful results. We discovered that every time they yielded the max PSNR for the following selected parameter values shown in Figures 18 and 19.

(ii) Power. We can claim our design to be energy efficient as the power consumption for the design has reduced in comparison to other benchmark works for an example image of a given resolution as shown (cf. Table 3 by reducing the number of computations [26], NB also tested for images of various resolutions) with respect to other state-of-the-art works cited previously $[11,12]$. 

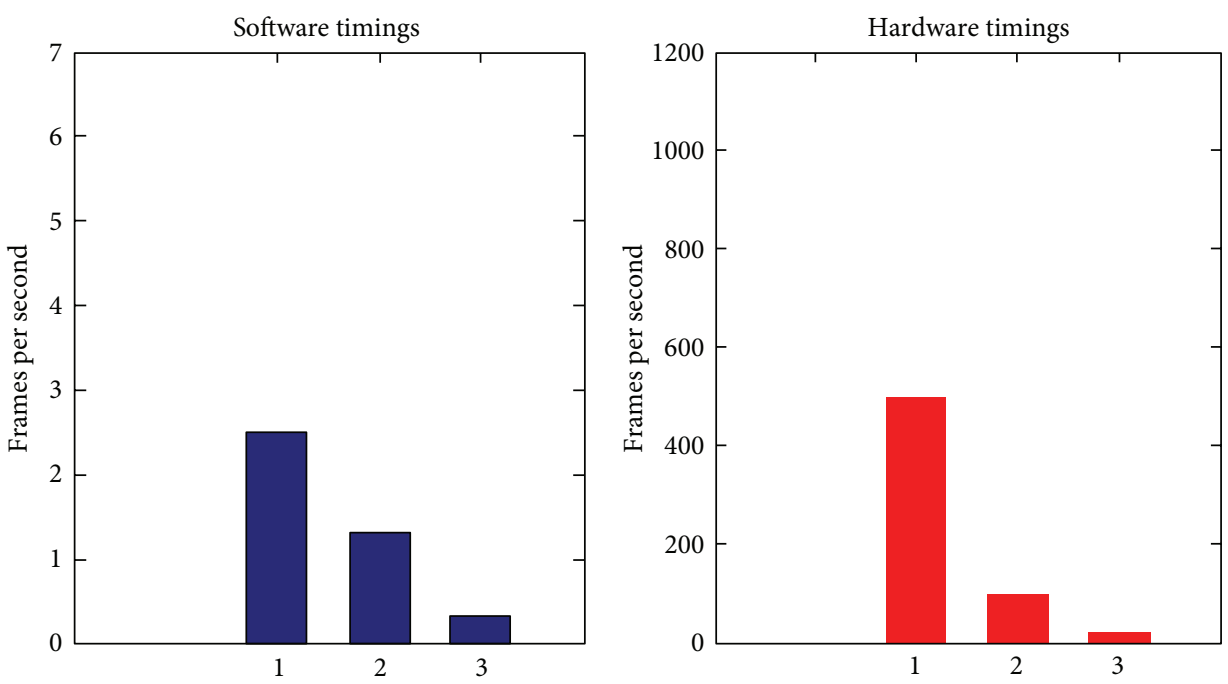

Image resolution
(1) $256 \times 256$
(2) $512 \times 512$
(3) $1024 \times 1024$

FIGURE 22: The figure shows the frame processing rate of software and hardware processing platform. $x$-axis denotes the image resolutions and $y$-axis the frames per second, respectively, for image of different resolutions as shown from 1 to 3 in $x$-axis.

TABLE 4: Comparison showing resource utilization of the various optimized hardware architectures for image resolution $150 \times 150$ implemented in Virtex-5 LX110T OpenSPARC Evaluation Platform [28] and Spartan 6 IVK [29] realizing bilateral [12] and anisotropic diffusion filtering.

\begin{tabular}{|c|c|c|c|}
\hline \multirow[b]{2}{*}{ Percentage utilization } & \multicolumn{3}{|c|}{ Image size $(150 \times 150)$} \\
\hline & $\begin{array}{l}\text { Virtex-5 LX110T } \\
\text { OpenSPARC FPGA } \\
\text { (utilized/total number) } \\
\text { (anisotropic diffusion) }\end{array}$ & $\begin{array}{l}\text { Fully parallel and separable } \\
\text { single dimensional architecture } \\
\text { (bilateral filter) for the same } \\
\text { OpenSPARC device }\end{array}$ & $\begin{array}{c}\text { Avnet Spartan } 6 \\
\text { industrial video } \\
\text { processing kit } \\
\text { (anisotropic diffusion) }\end{array}$ \\
\hline \multirow{2}{*}{ Occupied slices } & $5225 / 17280$ & 6342 and 3144 & $3810 / 23038$ \\
\hline & $(30 \%)$ & $(37 \%$ and $18 \%)$ & $(16 \%)$ \\
\hline \multirow{2}{*}{ Slice LUTs } & $14452 / 69120$ & 11689 and 8535 & $11552 / 92152$ \\
\hline & $(20 \%)$ & $(17 \%$ and $12 \%)$ & $(12 \%)$ \\
\hline \multirow{2}{*}{ Block-RAM/FIFO/RAMB8BWERs } & $1 / 148$ & 22 and 22 & $2 / 536$ \\
\hline & $(1 \%)$ & $(15 \%$ and $15 \%)$ & $(1 \%)$ \\
\hline \multirow{2}{*}{ Flip flops } & $17309 / 69120$ & 16167 and 5440 & $15214 / 69120$ \\
\hline & $(25 \%)$ & $(23 \%$ and $8 \%)$ & $(22 \%)$ \\
\hline \multirow{2}{*}{ Bonded IOBs } & $46 / 640$ & 1 and 1 & $46 / 396$ \\
\hline & $(7 \%)$ & $(1 \%$ and $1 \%)$ & $(11 \%)$ \\
\hline \multirow{2}{*}{ Mults/DSP48Es/DSP48A1s } & $55 / 64$ & 0 and 0 & $81 / 180$ \\
\hline & $(85 \%)$ & $(0 \%$ and $0 \%)$ & $(45 \%)$ \\
\hline \multirow{2}{*}{ BUFGs/BUFCTRLs } & $1 / 32$ & 4 and 4 & 1 \\
\hline & $(3 \%)$ & $(13 \%$ and $13 \%)$ & $(3 \%)$ \\
\hline
\end{tabular}

(iii) Diffusion Coefficient Analysis. We performed some performance analysis on the diffusion coefficient responsible for controlling the filtering process, subsequently by differentiation and scaling. The changes in the signal behavior help to perform a proper selection of the scaling parameter needed for filtering different image types. PSNR and SSIM performance measures reflect the reconstructed denoising quality affected by random noise.

(iv) Complexity. Previous implementations [10, 15] used normal convolution operation to calculate the intensity gradients whose computational complexity is of 
TABLE 5: Runtime comparison in software and hardware for bilateral filtering (BF) and anisotropic diffusion (AD) filtering (note that $\mathrm{A}=150$ $\times 150, B=256 \times 256, C=512 \times 512$, and $\mathrm{D}=1024 \times 1024)$. The processing platform was done on an Intel(R) Core(TM) 2 Duo CPU T6600 $3.2 \mathrm{GHz}$ system.

\begin{tabular}{|c|c|c|c|c|c|c|c|c|}
\hline \multirow{2}{*}{$\begin{array}{l}\text { Filtering techniques } \\
\text { Image resolution }\end{array}$} & \multicolumn{4}{|c|}{ AD filtering } & \multicolumn{4}{|c|}{$\mathrm{BF}$} \\
\hline & $\mathrm{A}$ & $\mathrm{B}$ & C & $\mathrm{D}$ & $\mathrm{A}$ & $\mathrm{B}$ & $\mathrm{C}$ & $\mathrm{D}$ \\
\hline $\begin{array}{l}\text { Execution time (software in } \\
\text { seconds) }\end{array}$ & 0.101 & 0.153 & 0.402 & 1.1 & 0.5 & 1.1 & 2.5 & 11.5 \\
\hline $\begin{array}{l}\text { Acceleration rate in software for } \\
\text { anisotropic over bilateral (approx.) }\end{array}$ & $3 x$ & $3 x$ & $3 x$ & $3 x$ & - & - & - & - \\
\hline $\begin{array}{l}\text { Acceleration rate when executed in } \\
\text { hardware with respect to software } \\
\text { for } \mathrm{BF}\end{array}$ & - & - & - & - & $70 x$ & $6 x$ & $7 x$ & $3 \mathrm{x}$ \\
\hline $\begin{array}{l}\text { Acceleration rate when executed in } \\
\text { hardware with respect to software } \\
\text { for } \mathrm{AD}\end{array}$ & $91 x$ & $46 x$ & $30 x$ & $21 x$ & - & - & - & - \\
\hline
\end{tabular}

TABLE 6: Complexity analysis report. The set $S$ of all possible image locations. The set $R$ of all possible pixel values. $\sigma$ is the kernel standard deviation. $M \times N$ denotes the image resolution, $x$ is patch size, and $y$ is the search window size.

\begin{tabular}{lc}
\hline Algorithm & Complexity \\
\hline $\begin{array}{l}\text { Constant time polynomial range } \\
\text { approach [30] }\end{array}$ & $O(1)$ \\
Trilateral filter [13] & More than the \\
NLM [27] & {$[12,27,30-35]$} \\
Brut force approach [31] & $x^{2} \cdot y^{2} \cdot N \cdot M$ \\
Layered approach [32] & $O\left(|S|^{2}\right)$ \\
Bilateral grid [33] approach & $O\left(|S|+\left(|S| / \sigma_{s}^{2}\right)\left(|R| / \sigma_{r}\right)\right)$ \\
Separable filter kernel approach [34] & $O\left(|S|+\left(|S| / \sigma_{s}^{2}\right)\left(|R| / \sigma_{r}\right)\right)$ \\
Local histogram approach [35] & $O\left(|S| \sigma_{s}\right)$ \\
Constant time trigonometric range & $O\left(|S| \log \sigma_{s}\right)$ \\
approach [12] & $O(1)$ \\
Classical anisotropic diffusion [2] & Nonlinear \\
$\begin{array}{l}\text { Optimized anisotropic diffusion }(O A D) \\
\text { (our approach) }\end{array}$ & $O(1)$ \\
\hline
\end{tabular}

$O\left(N^{2}\right)$. Even if the normal convolution is substituted by single dimensional architecture [12], the computational complexity would reduce to $O(N)$. However, we have realized the same with a single arithmetic subtraction operation, making it convenient by arranging the pixels in the favorable order, thereby reducing the complexity to $O(1)$. That is, $O\left(N^{2}\right) \rightarrow$ $O(N) \rightarrow O(1)$, that is, the least complexity achievable.

(v) Speed. Besides having $O(1)$ complexity, our hardware architecture of the algorithm has been formulated in parallel. This allows us to further accelerate its speed, since all the directional gradient computations have been done in parallel, thereby saving the CORDIC (processor) divider delay time by $(41 * 7 *$ 10) $=2870$ ns. Each CORDIC block has 31-unit delay, together with multipliers and registers and thereby saving 7 directions (due to parallel executing) where $10 \mathrm{~ns}$ is each clock pulse.

(vi) Adaptive Iteration. We have designed Algorithm 1, which shows the steps of intelligent adaptation of the number of iterations.

(vii) The filter design has been implemented in one grayscale channel; however, it can be replicated for all other color channels.

(viii) Reconstruction Quality. Last but not least, the denoised image quality has been measured against benchmark quality performance metrics.

\section{Conclusions}

In this paper, we presented an efficient hardware implementation of edge-preserving anisotropic diffusion filter. Considerable gain with respect to accuracy, power, complexity, speed, and reconstruction quality has been obtained as discussed in Section 6. Our design has been compared to the hardware implementation of state-of-the-art works with respect to acceleration, energy consumption, PSNR, SSIM, and so forth. From the point of view of the hardware realization of edgepreserving filters, both bilateral and anisotropic diffusion yield satisfying results, but still the research community prefers bilateral filter as it has less parameters to tune and is noniterative in nature. However, recent implementations of the same are iterative for achieving higher denoised image quality. So it can be concluded that if a proper selection of parameters can be done (has been made adaptive without manual intervention in our case) in case of anisotropic diffusion filtering, then real-time constraints can be overcome without much overhead. We have not performed the hardware implementation of the nonlocal means algorithm as it contains exponential operations at every step. Hardware implementation of the exponential operation introduces a lot of approximation errors.

While GPU implementations of the same do exist, however, we have undertaken this work as a case study to measure the hardware performance of the same. 
Additional work on testing with more images, design optimization, and real-time demonstration of the system and a suitable physical design (floorplanning to masking) is to be carried out in future. It is to be noted that we have designed one extended trilateral filter algorithm (edge-preserving/denoising) which is also producing promising results (not been published yet).

Till now, there have been more advanced versions of anisotropic diffusion algorithms even with more optimized/ modified versions. But they are all optimized and targeted to specific applications. However, this design forms the base architecture for all the other designs. Any kind of modification of the algorithm and its corresponding hardware design can be done keeping the similar base architecture.

\section{Competing Interests}

The authors declare that there are no competing interests regarding the publication of this paper.

\section{Acknowledgments}

This work has been supported by the Department of Science and Technology, Government of India, under Grant no. DST/INSPIRE FELLOWSHIP/2012/320 as well as the grant from TEQIP phase 2 (COE) of the University of Calcutta providing fund for this research. The authors also wish to thank Mr. Pabitra Das and Dr. Kunal Narayan Choudhury for their valuable suggestions.

\section{References}

[1] W. Wu, S. T. Acton, and J. Lach, "Real-time processing of ultrasound images with speckle reducing anisotropic diffusion," in Proceedings of the 40th Asilomar Conference on Signals, Systems, and Computers (ACSSC '06), pp. 1458-1464, Pacific Grove, Calif, USA, November 2006.

[2] P. Perona and J. Malik, "Scale-space and edge detection using anisotropic diffusion," IEEE Transactions on Pattern Analysis and Machine Intelligence, vol. 12, no. 7, pp. 629-639, 1990.

[3] D. Bailey, "Implementing machine vision systems using FPGAs," in Machine Vision Handbook, B. G. Batchelor, Ed., pp. 1103-1136, Springer, London, UK, 2012.

[4] R. Zatrepalek, Using FPGAs to Solve Tough DSP Design Challenges, 2007, http://www.eetimes.com/document.asp?piddl_ msgpage $=2 \& d o c \_i d=1279776 \&$ page_number $=1$.

[5] J. A. Kalomiros and J. Lygouras, "Design and evaluation of a hardware/software FPGA-based system for fast image processing," Microprocessors and Microsystems, vol. 32, no. 2, pp. 95106, 2008.

[6] A. E. Nelson, "Implementation of image processing algorithms on FPGA hardware," May 2000, http://www.isis.vanderbilt.edu/ sites/default/files/Nelson_T_0_0_2000_Implementa.pdf.

[7] B. G. Batchelor, Machine Vision Handbook, Springer, London, UK, 2012.

[8] K. T. Gribbon, D. G. Bailey, and C. T. Johnston, "Design patterns for image processing algorithm development on FPGAs," in Proceedings of the IEEE Region 10 Conference (TENCON '05), pp. 1-6, Melbourne, Australia, November 2005.
[9] S.-K. Han, M.-H. Jeong, S. Woo, and B.-J. You, "Architecture and implementation of real-time stereo vision with bilateral background subtraction," in Advanced Intelligent Computing Theories and Applications. With Aspects of Theoretical and Methodological Issues, D.-S. Huang, L. Heutte, and M. Loog, Eds., vol. 4681 of Lecture Notes in Computer Science, pp. 906912, Springer, Berlin, Germany, 2007.

[10] W. Atabany and P. Degenaar, "Parallelism to reduce power consumption on FPGA spatiotemporal image processing," in Proceedings of the IEEE International Symposium on Circuits and Systems (ISCAS '08), pp. 1476-1479, Seattle, Wash, USA, May 2008.

[11] K. N. Chaudhury, D. Sage, and M. Unser, "Fast O(1) bilateral filtering using trigonometric range kernels," IEEE Transactions on Image Processing, vol. 20, no. 12, pp. 3376-3382, 2011.

[12] C. Pal, K. N. Chaudhury, A. Samanta, A. Chakrabarti, and R. Ghosh, "Hardware software co-design of a fast bilateral filter in FPGA," in Proceedings of the 10th Annual Conference of the IEEE India Council (INDICON '13), pp. 1-6, Mumbai, India, December 2013.

[13] P. Choudhury and J. Tumblin, "The trilateral filter for high contrast images and meshes," in Proceedings of the 14th Eurographics Symposium on Rendering, pp. 186-196, 2003.

[14] O. Dandekar, C. Castro-Pareja, and R. Shekhar, "FPGA-based real-time $3 \mathrm{D}$ image preprocessing for image-guided medical interventions," Journal of Real-Time Image Processing, vol. 1, no. 4, pp. 285-301, 2007.

[15] D. Bera and S. Banerjee, "Pipelined DSP implementation of nonlinear anisotropic diffusion for speckle reduction of USG images," in Proceedings of the 2nd International Conference on Computer Engineering and Technology (ICCET '10), pp. V249V253, Chengdu, China, April 2010.

[16] M. Howison, "Comparing GPU implementations of bilateral and anisotropic diffusion filters for 3D biomedical datasets," Tech. Rep., 2010.

[17] S. Che, J. Li, J. W. Sheaffer, K. Skadron, and J. Lach, "Accelerating compute-intensive applications with GPUs and FPGAs," in Proceedings of the Symposium on Application Specific Processors (SASP '08), pp. 101-107, Anaheim, Calif, USA, June 2008.

[18] R. C. Gonzalez and E. Richard, Woods, Digital Image Processing, Pearson, 3rd edition, 2008.

[19] D. Lopes, Anisotropic Diffusion (Perona \& Malik), 2007, http:// www.mathworks.in/matlabcentral/fileexchange/14995-anisotropic-diffusion-perona-malik.

[20] I. Bravo, P. Jiménez, M. Mazo, J. L. Lázaro, and E. Martín, "Architecture based on FPGA's for real-time image processing," in Reconfigurable Computing: Architectures and Applications: Second International Workshop, ARC 2006, Delft, The Netherlands, March 1-3, 2006, Revised Selected Papers, vol. 3985 of Lecture Notes in Computer Science, pp. 152-157, Springer, Berlin, Germany, 2006.

[21] K. P. Sarawadekar, H. B. Indana, D. Bera, and S. Banerjee, "VLSIDSP based real time solution of DSC-SRI for an ultrasound system," Microprocessors and Microsystems, vol. 36, no. 1, pp. 1$12,2012$.

[22] S. McBader and P. Lee, "An FPGA implementation of a flexible, parallel image processing architecture suitable for embedded vision systems," in Proceedings of the International Parallel and Distributed Processing Symposium (IPDPS '03), pp. 1-5, Nice, France, April 2003.

[23] http://www.mathworks.com/products/hdl-verifier. 
[24] System Generator for DSP Getting Started Guide. Release 10.1, March 2008.

[25] A. T. Moreo, P. N. Lorente, F. S. Valles, J. S. Muro, and C. F. Andrés, "Experiences on developing computer vision hardware algorithms using Xilinx system generator," Microprocessors and Microsystems, vol. 29, no. 8-9, pp. 411-419, 2005.

[26] Xilinx Power Tools Tutorial, Spartan-6 and Virtex-6 FPGAs [Optional] UG733 (v13.1), 2011.

[27] A. Buades, B. Coll, and J. M. Morel, "Denoising image sequences does not require motion estimation," in Proceedings of the IEEE Conference on Advanced Video and Signal Based Surveillance (AVSS '05), pp. 70-74, Como, Italy, September 2005.

[28] Virtex-5 OpenSPARC Evaluation Platform (ML509), http:// www.digilentinc.com/Products/Detail.cfm?Prod=XUPV5.

[29] Xilinx Spartan-6 FPGA Industrial Video Processing Kit, http:// www.em.avnet.com/en-us/design/drc/Pages/Xilinx-Spartan-6FPGA-Industrial-Video-Processing-Kit.aspx.

[30] F. Porikli, "Constant time O(1) bilateral filtering," in Proceedings of the IEEE Conference on Computer Vision and Pattern Recognition (CVPR '08), pp. 1-8, IEEE, Anchorage, Alaska, June 2008.

[31] M. Elad, "On the origin of the bilateral filter and ways to improve it," IEEE Transactions on Image Processing, vol. 11, no. 10, pp. 1141-1151, 2002.

[32] F. Durand and J. Dorsey, "Fast bilateral filtering for the display of high-dynamic-range images," ACM Siggraph, vol. 21, no. 3, pp. 257-266, 2002.

[33] J. Chen, S. Paris, and F. Durand, "Real-time edge-aware image processing with the bilateral grid," in Special Interest Group on Computer Graphics and Interactive Techniques Conference (SIGGRAPH '07 ), ACM, New York, NY, USA, 2007.

[34] T. Q. Pham and L. J. van Vliet, "Separable bilateral filtering for fast video preprocessing," in IEEE International Conference on Multimedia and Expo (ICME '05), pp. 454-457, July 2005.

[35] B. Weiss, "Fast median and bilateral filtering," ACM Siggraph, vol. 25, no. 3, pp. 519-526, 2006. 


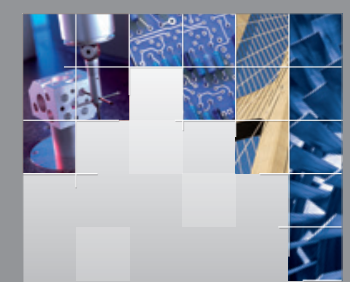

\section{Enfincering}
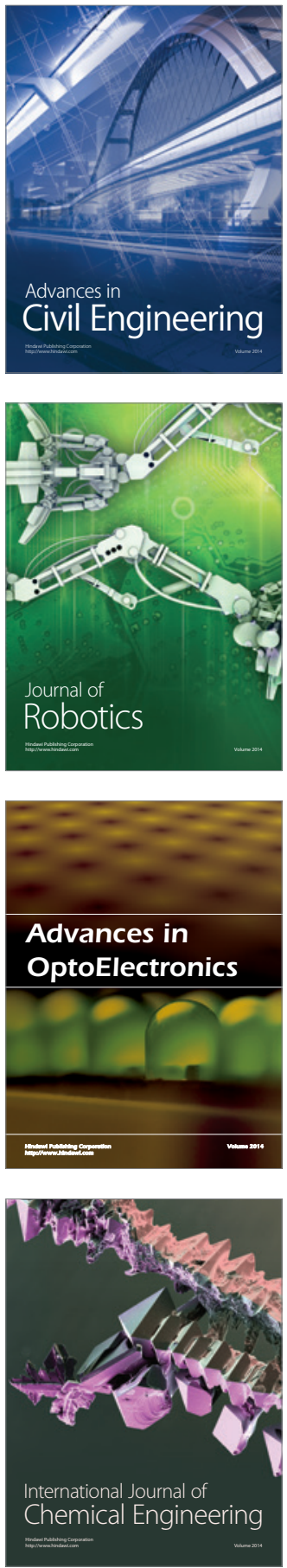

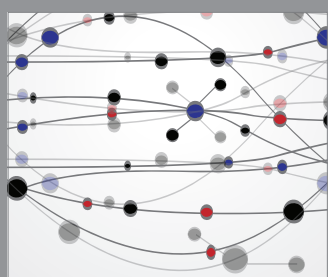

The Scientific World Journal

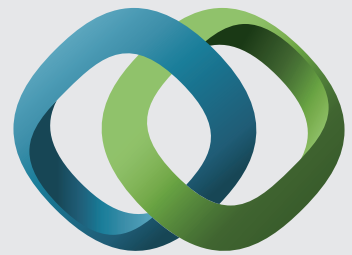

\section{Hindawi}

Submit your manuscripts at

http://www.hindawi.com
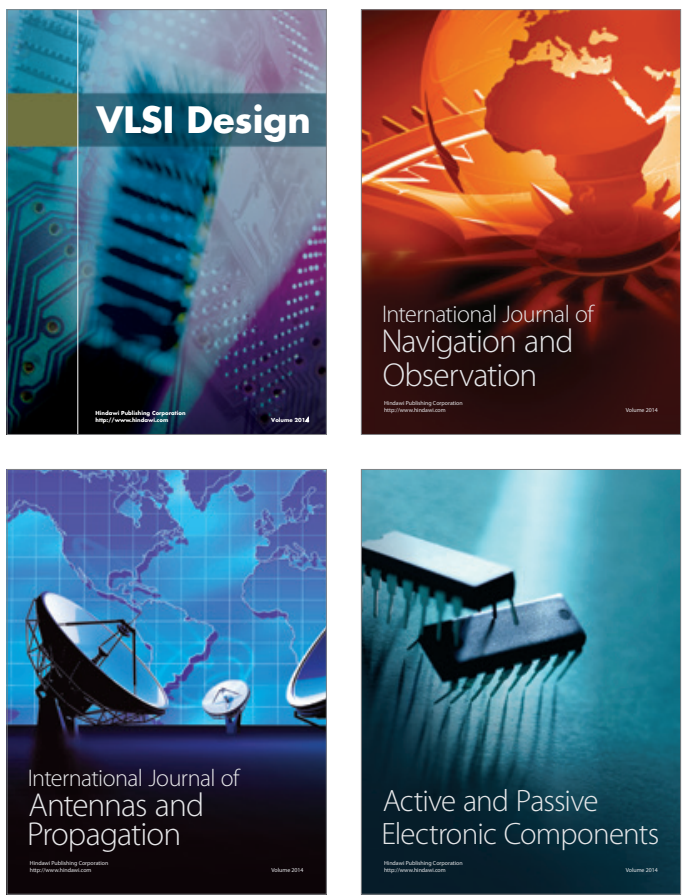
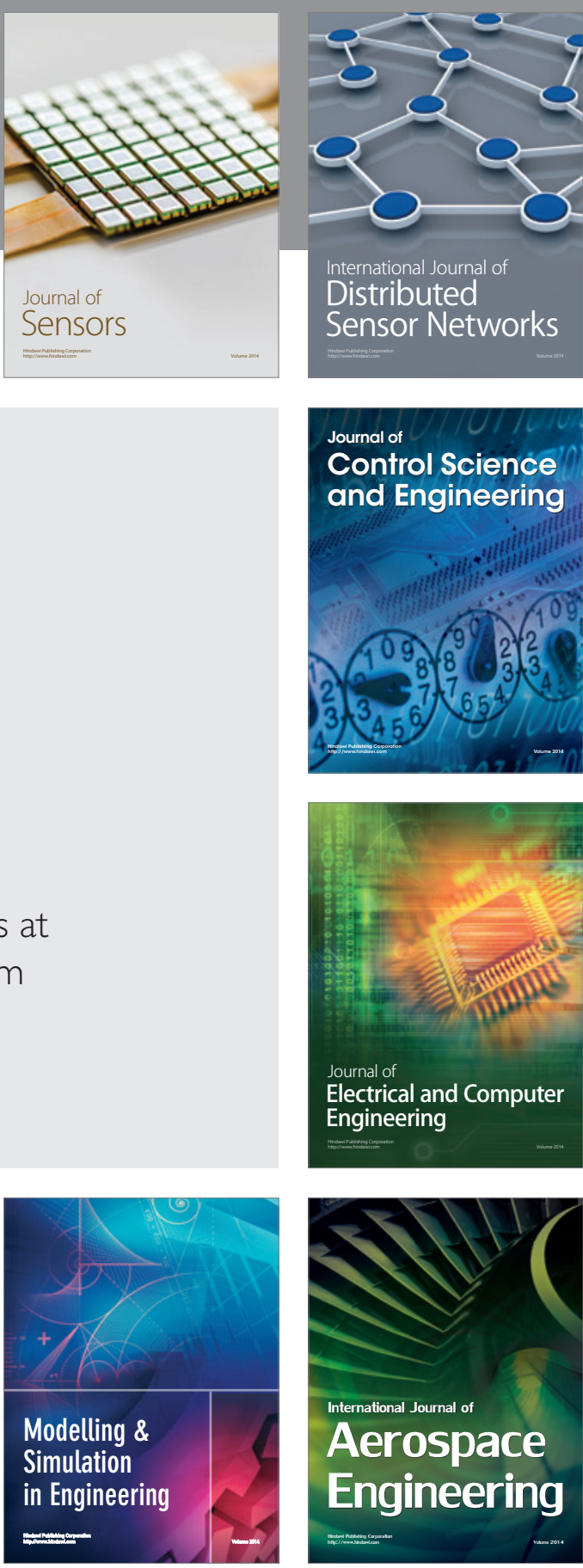

International Journal of

Distributed

Sensor Networks

Journal of

Control Science

and Engineering
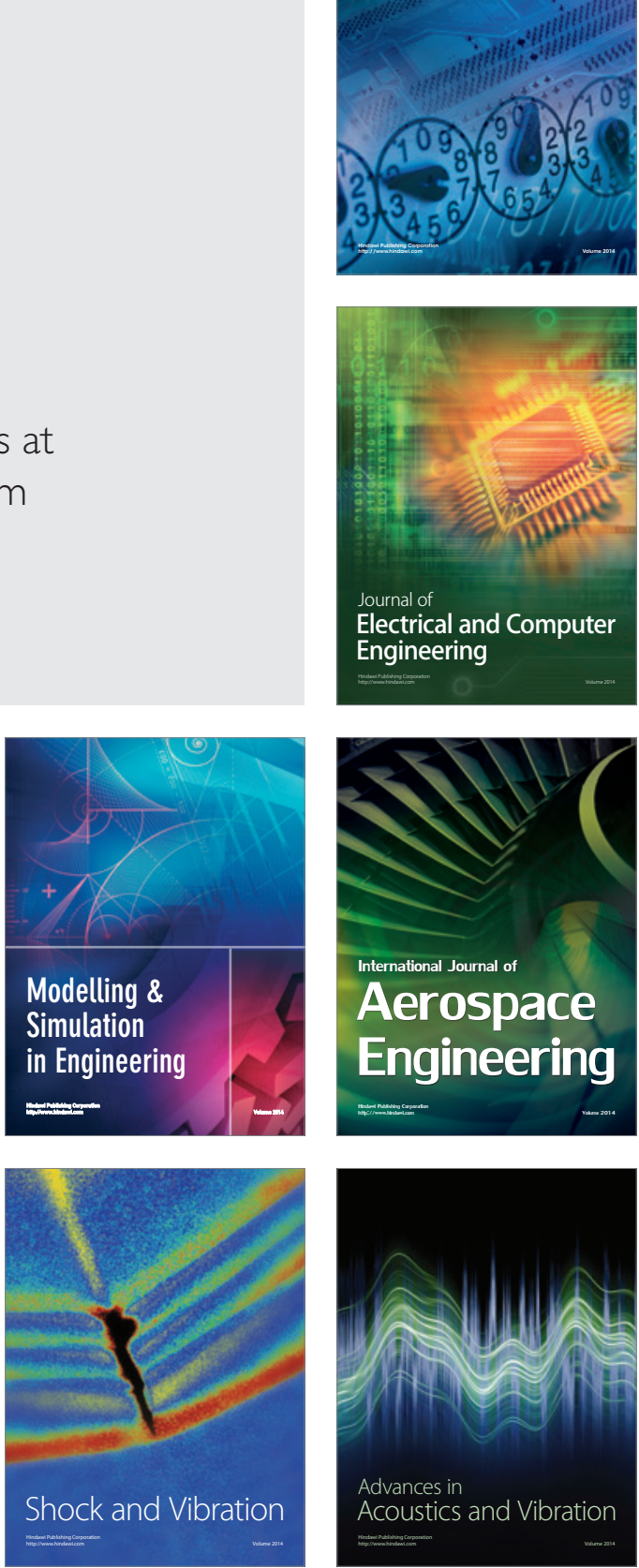